\title{
Hopf Bifurcation Analysis for a Four-Dimensional Recurrent Neural Network with Two Delays
}

\author{
Zizhen Zhang ${ }^{1,2}$ and Huizhong Yang ${ }^{1}$ \\ ${ }^{1}$ Key Laboratory of Advanced Process Control for Light Industry (Ministry of Education), Jiangnan University, Wuxi 214122, China \\ ${ }^{2}$ School of Management Science and Engineering, Anhui University of Finance and Economics, Bengbu 233030, China \\ Correspondence should be addressed to Huizhong Yang; yhz@jiangnan.edu.cn
}

Received 22 July 2013; Accepted 14 October 2013

Academic Editor: Carlos J. S. Alves

Copyright (c) 2013 Z. Zhang and H. Yang. This is an open access article distributed under the Creative Commons Attribution License, which permits unrestricted use, distribution, and reproduction in any medium, provided the original work is properly cited.

A four-dimensional recurrent neural network with two delays is considered. The main result is given in terms of local stability and Hopf bifurcation. Sufficient conditions for local stability of the zero equilibrium and existence of the Hopf bifurcation with respect to both delays are obtained by analyzing the distribution of the roots of the associated characteristic equation. In particular, explicit formulae for determining the direction of the Hopf bifurcation and the stability of the bifurcating periodic solutions are established by using the normal form theory and center manifold theory. Some numerical examples are also presented to verify the theoretical analysis.

\section{Introduction}

In recent years, neural networks have attracted many scholars' attention all over the world and have been applied in different areas such as signal processing [1], pattern recognition [2-4], optimization [5], and automatic control [6-8]. In particular, the appearance of a cycle bifurcating from an equilibrium of an ordinary or a delayed neural network with a single parameter has been widely investigated [9-17]. In [18], Ruiz et al. studied the following recurrent neural network for the first time:

$$
\begin{gathered}
\dot{x}_{1}(t)=-x_{1}(t)+f\left(x_{2}(t)\right), \\
\vdots \\
\dot{x}_{n-1}=-x_{n-1}(t)+u(t), \\
\dot{x}_{n}=-x_{n}(t)+w_{1} f\left(x_{1}(t)\right)+\cdots+w_{n-1} f\left(x_{n-1}(t)\right), \\
y(t)=f\left(x_{n}(t)\right),
\end{gathered}
$$

where $x(t) \in R^{n}$ is the state, $w_{i} \in R, i=1, \ldots, n-1$ are the network parameters or weights, $u(t)$ is the input, $y(t)$ is the output, and $f(\cdot)$ is the transfer function of the neurons. The three-node network of system (1) in the feedback configuration, with $u(t)=y(t)$, has been studied in $[12,18,19]$; that is

$$
\begin{aligned}
& \dot{x}_{1}(t)=-x_{1}(t)+f\left(x_{2}(t)\right), \\
& \dot{x}_{2}(t)=-x_{2}(t)+f\left(x_{3}(t)\right), \\
& \dot{x}_{3}(t)=-x_{3}(t)+w_{1} f\left(x_{1}(t)\right)+w_{2} f\left(x_{2}(t)\right) .
\end{aligned}
$$

It is well known that time delays can play a complicated role on neural networks. They can be the source of instabilities and bifurcation in neural networks. Based on this fact, Hajihosseini et al. [11] considered system (2) with distributed delays and $f(\cdot)=\tanh (\cdot)$. It is shown that a Hopf bifurcation takes place in the delayed system as the mean delay passes a critical value where a family of periodic solutions bifurcate from the equilibrium. The existence and stability of such solutions are determined by the Hopf bifurcation theorem in the frequency domain and the generalized Nyquist stability criterion.

As far as we know, there are some papers on the bifurcations of neural network with two or multiple delays [20-22]. Motivated by the work in [11, 20-22] and considering that when the number of neurons is large, the simplified model 
can reflect the really large neural networks more closely, we consider the following four-dimensional recurrent neural network with two discrete delays that occur in the interaction between the neurons:

$$
\begin{aligned}
\dot{x}_{1}(t)= & -x_{1}(t)+f\left(x_{2}\left(t-\tau_{1}\right)\right), \\
\dot{x}_{2}(t)= & -x_{2}(t)+f\left(x_{3}\left(t-\tau_{1}\right)\right), \\
\dot{x}_{3}(t)= & -x_{3}(t)+f\left(x_{4}\left(t-\tau_{1}\right)\right), \\
\dot{x}_{4}(t)= & -x_{4}(t)+w_{1} f\left(x_{1}\left(t-\tau_{2}\right)\right) \\
& +w_{2} f\left(x_{2}\left(t-\tau_{2}\right)\right)+w_{3} f\left(x_{3}\left(t-\tau_{2}\right)\right),
\end{aligned}
$$

where $\tau_{1} \geq 0, \tau_{2} \geq 0$ are time delays that occur in the interaction between the neurons.

This paper is organized as follows. In Section 2, the stability of the zero equilibrium of system (3) and the existence of local Hopf bifurcation with respect to possible combinations of the two delays are investigated. In Section 3, the properties of the Hopf bifurcation such as the direction and the stability are determined by using the normal form theory and center manifold theory. Some numerical simulations are also included in Section 4 to illustrate the validity of the main results.

\section{Stability of the Zero Equilibrium and Local Hopf Bifurcation}

Throughout this paper we make the following assumption on the transfer function $f(\cdot)$ :

(H) $f \in C^{4}(R), f(0)=0$, and $f^{\prime}(0) \neq 0$.

Clearly, $E_{0}=(0,0,0,0)^{T}$ is the zero equilibrium of system (3). Linearization of system (3) at the zero equilibrium is

$$
\begin{aligned}
\dot{x}_{1}(t)= & -x_{1}(t)+f^{\prime}(0) x_{2}\left(t-\tau_{1}\right), \\
\dot{x}_{2}(t)= & -x_{2}(t)+f^{\prime}(0) x_{3}\left(t-\tau_{1}\right), \\
\dot{x}_{3}(t)= & -x_{3}(t)+f^{\prime}(0) x_{4}\left(t-\tau_{1}\right), \\
\dot{x}_{4}(t)= & -x_{4}(t)+w_{1} f^{\prime}(0) x_{1}\left(t-\tau_{2}\right) \\
& +w_{2} f^{\prime}(0) x_{2}\left(t-\tau_{2}\right)+w_{3} f^{\prime}(0) x_{3}\left(t-\tau_{2}\right) .
\end{aligned}
$$

The characteristic equation of the linearized system (4) is

$$
\begin{gathered}
(\lambda+1)^{4}+A(\lambda+1)^{2} e^{-\lambda\left(\tau_{1}+\tau_{2}\right)}+B(\lambda+1) e^{-\lambda\left(2 \tau_{1}+\tau_{2}\right)} \\
+C e^{-\lambda\left(3 \tau_{1}+\tau_{2}\right)}=0,
\end{gathered}
$$

where

$$
A=-w_{3} f^{\prime 2}(0), \quad B=-w_{2} f^{\prime 3}(0), \quad C=-w_{1} f^{\prime 4}(0) .
$$

In order to study the local stability of the zero equilibrium of system (3), we investigate the distribution of the roots of (5) in the following.
Case $1\left(\tau_{1}=\tau_{2}=0\right)$. Equation (5) reduces to

$$
\lambda^{4}+d_{1} \lambda^{3}+d_{2} \lambda^{2}+d_{3} \lambda+d_{4}=0
$$

where

$$
\begin{gathered}
d_{1}=4, \quad d_{2}=A+6, \quad d_{3}=2 A+B+4, \\
d_{4}=B+C+1 .
\end{gathered}
$$

Obviously, $D_{1}=d_{1}>0$. Therefore, by the Routh-Hurwitz criterion, the zero equilibrium $E_{0}(0,0,0,0)^{T}$ of system (3) is locally asymptotically stable if the following condition $\left(H_{1}\right)$ holds:

$$
\begin{gathered}
D_{2}=\left(\begin{array}{ll}
d_{1} & 1 \\
d_{3} & d_{2}
\end{array}\right)>0, \quad D_{3}=\left(\begin{array}{lll}
d_{1} & 1 & 0 \\
d_{3} & d_{2} & d_{1} \\
0 & d_{4} & d_{3}
\end{array}\right)>0, \\
D_{4}=\left(\begin{array}{cccc}
d_{1} & 1 & 0 & 0 \\
d_{3} & d_{2} & d_{1} & 1 \\
0 & d_{4} & d_{3} & d_{2} \\
0 & 0 & 0 & d_{4}
\end{array}\right)>0 .
\end{gathered}
$$

Case $2\left(\tau_{1}>0, \tau_{2}=0\right)$. On substituting $\tau_{2}=0$, (5) becomes

$$
(\lambda+1)^{4}+A(\lambda+1)^{2} e^{-\lambda \tau_{1}}+B(\lambda+1) e^{-2 \lambda \tau_{1}}+C e^{-3 \lambda \tau_{1}}=0 .
$$

Multiplying $e^{\lambda \tau_{1}}$ on both sides of (10), it is easy to obtain

$$
A(\lambda+1)^{2}+(\lambda+1)^{4} e^{\lambda \tau_{1}}+B(\lambda+1) e^{-\lambda \tau_{1}}+C e^{-2 \lambda \tau_{1}}=0 .
$$

Let $\lambda=i \omega_{1}\left(\omega_{1}>0\right)$ be a root of (11). Then, we can get

$$
\begin{aligned}
& A_{11} \cos \tau_{1} \omega_{1}+A_{12} \sin \tau_{1} \omega_{1}+A_{13}=A_{14}, \\
& A_{21} \cos \tau_{1} \omega_{1}+A_{22} \sin \tau_{1} \omega_{1}+A_{23}=A_{24},
\end{aligned}
$$

where

$$
\begin{gathered}
A_{11}=\omega_{1}^{4}-6 \omega_{1}^{2}+B-1, \quad A_{12}=4 \omega_{1}^{3}+B \omega_{1}-4 \omega_{1}, \\
A_{13}=A\left(1-\omega_{1}^{2}\right), \quad A_{14}=-C \cos 2 \tau_{1} \omega_{1}, \\
A_{21}=B \omega_{1}+4 \omega_{1}-4 \omega_{1}^{3}, \quad A_{22}=\omega_{1}^{4}-6 \omega_{1}^{2}-B+1, \\
A_{23}=2 A \omega_{1}, \quad A_{24}=C \sin 2 \tau_{1} \omega_{1} .
\end{gathered}
$$

Squaring both sides of the two equations in (12) and adding them up we obtain

$$
\begin{aligned}
& \left(A_{11} \cos \tau_{1} \omega_{1}+A_{12} \sin \tau_{1} \omega_{1}+A_{13}\right)^{2} \\
& \quad+\left(A_{21} \cos \tau_{1} \omega_{1}+A_{22} \sin \tau_{1} \omega_{1}+A_{23}\right)^{2}=C^{2} .
\end{aligned}
$$


According to $\sin \tau_{1} \omega_{1}= \pm \sqrt{1-\cos ^{2} \tau_{1} \omega_{1}}$, we consider the two cases.

(I) if $\sin \tau_{1} \omega_{1}=\sqrt{1-\cos ^{2} \tau_{1} \omega_{1}}$, then (14) takes the following form:

$$
\begin{aligned}
& \left(A_{11} \cos \tau_{1} \omega_{1}+A_{12} \sqrt{1-\cos ^{2} \tau_{1} \omega_{1}}+A_{13}\right)^{2} \\
& \quad+\left(A_{21} \cos \tau_{1} \omega_{1}+A_{22} \sqrt{1-\cos ^{2} \tau_{1} \omega_{1}}+A_{23}\right)^{2}=C^{2}
\end{aligned}
$$

which is equivalent to

$$
\begin{gathered}
p_{1} \cos ^{4} \tau_{1} \omega_{1}+p_{2} \cos ^{3} \tau_{1} \omega_{1}+p_{3} \cos ^{2} \tau_{1} \omega_{1} \\
+p_{4} \cos \tau_{1} \omega_{1}+p_{5}=0
\end{gathered}
$$

where

$$
\begin{aligned}
p_{1}= & \left(A_{11}^{2}+A_{21}^{2}-A_{12}^{2}-A_{22}^{2}\right)^{2}+4\left(A_{11} A_{12}+A_{21} A_{22}\right)^{2}, \\
p_{2}= & 4\left(A_{11}^{2}+A_{21}^{2}-A_{12}^{2}-A_{22}^{2}\right)\left(A_{11} A_{13}+A_{21} A_{23}\right) \\
& +8\left(A_{11} A_{12}+A_{21} A_{22}\right)\left(A_{12} A_{13}+A_{22} A_{23}\right), \\
p_{3}= & 4\left(A_{11} A_{13}+A_{21} A_{23}\right)^{2}+4\left(A_{12} A_{13}+A_{22} A_{23}\right)^{2} \\
& -4\left(A_{11} A_{12}+A_{21} A_{22}\right)^{2}+2\left(A_{11}^{2}+A_{21}^{2}-A_{12}^{2}-A_{22}^{2}\right) \\
& \times\left(A_{12}^{2}+A_{13}^{2}+A_{22}^{2}+A_{23}^{2}-C^{2}\right), \\
p_{4}= & 4\left(A_{11} A_{13}+A_{21} A_{23}\right)\left(A_{12}^{2}+A_{13}^{2}+A_{22}^{2}+A_{23}^{2}-C^{2}\right) \\
& -8\left(A_{11} A_{12}+A_{21} A_{22}\right)\left(A_{12} A_{13}+A_{22} A_{23}\right), \\
p_{5}= & \left(A_{12}^{2}+A_{13}^{2}+A_{22}^{2}+A_{23}^{2}-C^{2}\right)^{2} \\
& -\left(A_{12} A_{13}+A_{22} A_{23}\right)^{2} .
\end{aligned}
$$

Let $r=\cos \tau_{1} \omega_{1}$, and denote that

$$
f(r)=r^{4}+\frac{p_{2}}{p_{1}} r^{3}+\frac{p_{3}}{p_{1}} r^{2}+\frac{p_{4}}{p_{1}} r+\frac{p_{5}}{p_{1}}
$$

Thus,

$$
f^{\prime}(r)=4 r^{3}+\frac{3 p_{2}}{p_{1}} r^{2}+\frac{2 p_{3}}{p_{1}} r+\frac{p_{4}}{p_{1}}
$$

Let

$$
4 r^{3}+\frac{3 p_{2}}{p_{1}} r^{2}+\frac{2 p_{3}}{p_{1}} r+\frac{p_{4}}{p_{1}}=0
$$

Let $y=r+\left(p_{2} / 4 p_{1}\right)$. Then, (20) becomes

$$
y^{3}+\gamma_{1} y+\gamma_{0}=0
$$

where

$$
\gamma_{1}=\frac{p_{3}}{2 p_{1}}-\frac{3 p_{2}^{2}}{16 p_{1}^{2}}, \quad \gamma_{0}=\frac{p_{2}^{3}}{32 p_{1}^{3}}-\frac{p_{2} p_{3}}{8 p_{1}^{2}}+\frac{p_{4}}{4 p_{1}} .
$$

Define

$$
\beta_{1}=\left(\frac{\gamma_{2}}{2}\right)^{2}+\left(\frac{\gamma_{1}}{3}\right)^{3}, \quad \beta_{2}=\frac{-1+i \sqrt{3}}{2} .
$$

Then, we can get

$$
\begin{aligned}
& y_{1}=\sqrt[3]{-\frac{\gamma_{2}}{2}+\sqrt{\beta_{1}}}+\sqrt[3]{-\frac{\gamma_{2}}{2}-\sqrt{\beta_{1}}}, \\
& y_{2}=\sqrt[3]{-\frac{\gamma_{2}}{2}+\sqrt{\beta_{1}}} \beta_{2}+\sqrt[3]{-\frac{\gamma_{2}}{2}-\sqrt{\beta_{1}}} \bar{\beta}_{2}, \\
& y_{3}=\sqrt[3]{-\frac{\gamma_{2}}{2}+\sqrt{\beta_{1}}} \bar{\beta}_{2}+\sqrt[3]{-\frac{\gamma_{2}}{2}-\sqrt{\beta_{1}}} \beta_{2} .
\end{aligned}
$$

Then, we can get the expression of $\cos \tau_{1} \omega_{1}$ and we denote $f_{1}\left(\omega_{1}\right)=\cos \tau_{1} \omega_{1}$. Substituting $f_{1}\left(\omega_{1}\right)=\cos \tau_{1} \omega_{1}$ into (12), we can get the expression of $\sin \tau_{1} \omega_{1}$ and we denote $f_{2}\left(\omega_{1}\right)=\sin \tau_{1} \omega_{1}$. Thus, a function with respect to $\omega_{1}$ can be established by

$$
f_{1}^{2}\left(\omega_{1}\right)+f_{2}^{2}\left(\omega_{1}\right)=1 .
$$

We assume that $\left(H_{21}\right),(25)$, has finite positive roots, which are denoted by $\omega_{11}, \ldots, \omega_{1 k}$. For every fixed $\omega_{1 i}(1 \leq i \leq k)$, the corresponding critical value of time delay is

$$
\begin{aligned}
\tau_{1 i}^{(j)} & =\frac{1}{\omega_{1 i}} \arccos f_{1}\left(\omega_{1 i}\right)+\frac{2 j \pi}{\omega_{1 i}}, \\
& i=1,2, \ldots, k ; j=0,1,2, \ldots .
\end{aligned}
$$

Then, $\pm \omega_{1 i}$ are a pair of purely imaginary roots of (11) with $\tau_{1}=\tau_{1 i}^{(j)}$. Let

$$
\begin{array}{r}
\tau_{10}=\min \left\{\tau_{1 i}^{(j)}\right\}, \quad \omega_{10}=\omega_{1 i_{0}} \\
i=1,2, \ldots, k, j=0,1,2 \ldots
\end{array}
$$

(II) If $\sin \tau_{1} \omega_{1}=-\sqrt{1-\cos ^{2} \tau_{1} \omega_{1}}$, then (14) can be transformed into the following form:

$$
\begin{aligned}
& \left(A_{11} \cos \tau_{1} \omega_{1}-A_{12} \sqrt{1-\cos ^{2} \tau_{1} \omega_{1}}+A_{13}\right)^{2} \\
& \quad+\left(A_{21} \cos \tau_{1} \omega_{1}-A_{22} \sqrt{1-\cos ^{2} \tau_{1} \omega_{1}}+A_{23}\right)^{2}=C^{2} .
\end{aligned}
$$

Thus, similar as the process in case (I), we can get the expression of $\cos \tau_{1} \omega_{1}$ and $\sin \tau_{1} \omega_{1}$. Let

$$
f_{1 *}\left(\omega_{1}\right)=\cos \tau_{1} \omega_{1}, \quad f_{2 *}=\sin \tau_{1} \omega_{1} .
$$

Therefore,

$$
f_{1 *}^{2}\left(\omega_{1}\right)+f_{2 *}^{2}\left(\omega_{1}\right)=1 .
$$

Then, we can get the critical value of time delay corresponding to every fixed positive root $\omega_{1 i}^{\prime}$ of (30):

$$
\begin{gathered}
\tau_{1 i}^{(j)^{\prime}}=\frac{1}{\omega_{1 i}^{\prime}} \arccos f_{1}\left(\omega_{1 i}^{\prime}\right)+\frac{2 j \pi}{\omega_{1 i}^{\prime}}, \\
i=1,2, \ldots, k ; j=0,1,2 \ldots
\end{gathered}
$$


Let

$$
\begin{gathered}
\tau_{10}=\min \left\{\tau_{1 i}^{(j) \prime}\right\}, \quad \omega_{10}=\omega_{1 i_{0}}^{\prime}, \\
i=1,2, \ldots, k, \quad j=0,1,2, \ldots .
\end{gathered}
$$

Next, we verify the transversality. Taking the derivative of $\lambda$ with respect to $\tau_{1}$ in (11), we obtain

$$
\begin{aligned}
& {\left[\frac{d \lambda}{d \tau_{1}}\right]^{-1}} \\
& \quad=\frac{2 A(\lambda+1)+B e^{-\lambda \tau_{1}}+4(\lambda+1)^{3} e^{\lambda \tau_{1}}}{\lambda\left[(\lambda+1)^{4} e^{\lambda \tau_{1}}-B(\lambda+1) e^{-\lambda \tau_{1}}-2 C e^{-2 \lambda \tau_{1}}\right]}-\frac{\tau_{1}}{\lambda} .
\end{aligned}
$$

Thus,

$$
\operatorname{Re}\left[\frac{d \lambda}{d \tau_{1}}\right]_{\tau_{1}=\tau_{10}}^{-1}=\frac{P_{R} Q_{R}+P_{I} Q_{I}}{Q_{R}^{2}+Q_{I}^{2}}
$$

where

$$
\begin{aligned}
P_{R}= & \left(4-12 \omega_{10}^{2}+B\right) \cos \tau_{10} \omega_{10} \\
& -\left(3 \omega_{10}-\omega_{10}^{3}\right) \sin \tau_{10} \omega_{10}+2 A, \\
P_{I}= & \left(4-12 \omega_{10}^{2}-B\right) \sin \tau_{10} \omega_{10} \\
& +\left(3 \omega_{10}-\omega_{10}^{3}\right) \cos \tau_{10} \omega_{10}+2 A \omega_{10}, \\
Q_{R}= & \left(4 \omega_{10}^{4}+B \omega_{10}^{2}-4 \omega_{10}^{2}\right) \cos \tau_{10} \omega_{10} \\
& -\left(B \omega_{10}+\omega_{10}-6 \omega_{10}^{3}-\omega_{10}^{5}\right) \sin \tau_{10} \omega_{10} \\
& -2 C \omega_{10} \sin 2 \tau_{10} \omega_{10}, \\
Q_{I}= & \left(4 \omega_{10}^{4}-B \omega_{10}^{2}-4 \omega_{10}^{2}\right) \sin \tau_{10} \omega_{10} \\
& +\left(\omega_{10}-B \omega_{10}-6 \omega_{10}^{3}-\omega_{10}^{5}\right) \cos \tau_{10} \omega_{10} \\
& -2 C \omega_{10} \cos 2 \tau_{10} \omega_{10} .
\end{aligned}
$$

Obviously, if the condition $\left(H_{22}\right): P_{R} Q_{R}+P_{I} Q_{I} \neq 0$ holds, then $\operatorname{Re}\left[d \lambda / d \tau_{1}\right]_{\tau_{1}=\tau_{10}}^{-1} \neq 0$. Namely, if the condition $\left(H_{22}\right)$ holds, then the transversality condition is satisfied. By the discussion above and the Hopf bifurcation theorem in [23], it is easy to obtain the following results.

Theorem 1. If the condition $\left(H_{21}\right)$ means that (25) has finite positive roots and $\left(H_{22}\right)$ means that $P_{R} Q_{R}+P_{I} Q_{I} \neq 0$ holds, then the zero equilibrium $E_{0}$ of system (3) is asymptotically stable for $\tau_{1} \in\left[0, \tau_{10}\right)$, system (3) undergoes a Hopf bifurcation at $E_{0}$ when $\tau_{1}=\tau_{10}$, and a branch of periodic solutions bifurcates from the zero equilibrium near $\tau_{1}=\tau_{10}$.

Case $3\left(\tau_{2}>0, \tau_{1}=0\right)$. When $\tau_{1}=0$, (5) becomes the following form:

$$
(\lambda+1)^{4}+\left[A \lambda^{2}+(2 A+B) \lambda+A+B+C\right] e^{-\lambda \tau_{2}}=0 .
$$

Let $\lambda=i \omega_{2}\left(\omega_{2}>0\right)$ be a root of (36). Substituting it into (36) and separating the real and imaginary parts, we obtain

$$
\begin{aligned}
(2 A+ & B) \omega_{2} \sin \tau_{2} \omega_{2}+\left(A+B+C-A \omega_{2}^{2}\right) \cos \omega_{2} \tau_{2} \\
& =6 \omega_{2}^{2}-\omega_{2}^{4}-1, \\
(2 A+ & B) \omega_{2} \cos \tau_{2} \omega_{2}-\left(A+B+C-A \omega_{2}^{2}\right) \sin \omega_{2} \tau_{2} \\
& =4 \omega_{2}^{3}-4 \omega_{2} .
\end{aligned}
$$

It follows that

$$
\omega_{2}^{8}+c_{3} \omega_{2}^{6}+c_{2} \omega_{2}^{2}+c_{1} \omega_{2}+c_{0}=0,
$$

where

$$
\begin{gathered}
c_{0}=1-(A+B+C)^{2}, \quad c_{2}=6-A^{2}, \quad c_{3}=4 . \\
c_{1}=2 A(A+B+C)-(2 A+B)^{2}+4 .
\end{gathered}
$$

Let $\omega_{2}^{2}=z$, then (38) can be transformed into

$$
z^{4}+c_{3} z^{3}+c_{2} z^{2}+c_{1} z+c_{0}=0 .
$$

Next, we make the following assumption.

$\left(H_{31}\right)$ means that $(40)$ has at least one positive root.

Without loss of generality, we assume that (40) has four positive roots, which are denoted by $z_{1}, z_{2}, z_{3}$, and $z_{4}$. Thus, (38) has four positive roots $\omega_{2 k}=\sqrt{z_{k}}, k=1,2,3,4$. The corresponding critical value of time delay is

$$
\begin{aligned}
\tau_{2 k}^{(j)}=\frac{1}{\omega_{2 k}} \arccos \left(\left(A \omega_{2 k}^{6}+\right.\right. & (A+3 B-C) \omega_{2 k}^{4} \\
+ & \left.(2 B+6 C-A) \omega_{2 k}^{2}-(A+B+C)\right) \\
\times & \left((2 A+B)^{2} \omega_{2 k}^{2}\right. \\
+ & \left(A+B+C-A \omega_{2 k}^{2}\right)^{2} \\
& \left.\left.\times\left(6 \omega_{2 k}^{2}-\omega_{2 k}^{4}-1\right)^{2}\right)^{-1}\right)+\frac{2 j \pi}{\omega_{2 k}}, \\
& k=1,2,3,4 ; j=0,1,2 \ldots
\end{aligned}
$$

Then, $\pm i \omega_{2 k}$ are a pair of purely imaginary roots of (36) with $\tau_{2}=\tau_{2 k}^{(j)}$. Let

$$
\tau_{20}=\min \left\{\tau_{2 k}^{(0)}\right\}, \quad k=1,2,3,4, \quad \omega_{20}=\omega_{2 k_{0}} .
$$

Taking the derivative of $\lambda$ with respect to $\tau_{2}$ in (36), we can get

$$
\begin{aligned}
{\left[\frac{d \lambda}{d \tau_{2}}\right]^{-1}=} & -\frac{4 \lambda^{3}+12 \lambda^{2}+12 \lambda+4}{\lambda\left(\lambda^{4}+4 \lambda^{3}+6 \lambda^{2}+4 \lambda+1\right)} \\
& +\frac{2 A \lambda+2 A+B}{\lambda\left[A \lambda^{2}+(2 A+B) \lambda+A+B+C\right]}-\frac{\tau_{2}}{\lambda} .
\end{aligned}
$$


Then, we can get

$$
\begin{aligned}
\operatorname{Re}\left[\frac{d \lambda}{d \tau_{2}}\right]_{\tau=\tau_{20}}^{-1}= & \frac{4 \omega_{20}^{6}+12 \omega_{20}^{4}+12 \omega_{20}^{2}+4}{\omega_{20}^{8}+4 \omega_{20}^{6}+6 \omega_{20}^{4}+4 \omega_{20}^{2}+1} \\
- & \left(2 A^{2} \omega_{20}^{2}+(2 A+B)^{2}-2 A(A+B+C)\right) \\
& \times\left(A^{2} \omega_{20}^{4}+\left[(2 A+B)^{2}-2 A(A+B+C)\right]\right. \\
& \left.\times \omega_{20}^{2}+(A+B+C)^{2}\right)^{-1} .
\end{aligned}
$$

From (38), we have

$$
\begin{aligned}
\omega_{20}^{8}+ & 4 \omega_{20}^{6}+6 \omega_{20}^{4}+4 \omega_{20}^{2}+1 \\
= & A^{2} \omega_{20}^{4}+\left[(2 A+B)^{2}-2 A(A+B+C)\right] \omega_{20}^{2} \\
& +(A+B+C)^{2} .
\end{aligned}
$$

Thus,

$$
\operatorname{Re}\left[\frac{d \lambda}{d \tau_{2}}\right]_{\tau=\tau_{20}}^{-1}=\frac{g^{\prime}\left(z_{0}\right)}{\omega_{20}^{8}+4 \omega_{20}^{6}+6 \omega_{20}^{4}+4 \omega_{20}^{2}+1},
$$

where

$$
g(z)=z^{4}+c_{3} z^{3}+c_{2} z^{2}+c_{1} z+c_{0}, \quad z_{0}=\omega_{20}^{2} .
$$

Therefore, if the condition $\left(H_{32}\right): \quad g^{\prime}\left(z_{0}\right) \neq 0$, then $\operatorname{Re}\left[d \lambda / d \tau_{2}\right]_{\tau=\tau_{20}}^{-1} \neq 0$. From the analysis above and by the Hopf bifurcation theorem in [23], we have the following results.

Theorem 2. If the condition $\left(H_{31}\right)$ means that (40) has at least one positive root and $\left(H_{32}\right)$ means that $g^{\prime}\left(z_{0}\right) \neq 0$ holds, then the zero equilibrium $E_{0}$ of system (3) is asymptotically stable for $\tau_{2} \in\left[0, \tau_{20}\right)$, system (3) undergoes a Hopf bifurcation at $E_{0}$ when $\tau_{2}=\tau_{20}$, and a branch of periodic solutions bifurcates from the zero equilibrium near $\tau_{2}=\tau_{20}$.

Case $4\left(\tau_{1}=\tau_{2}=\tau>0\right)$. For $\tau_{1}=\tau_{2}=\tau>0$, (5) can be transformed into the following form:

$$
(\lambda+1)^{4}+A(\lambda+1)^{2} e^{-2 \lambda \tau}+B(\lambda+1) e^{-3 \lambda \tau}+C e^{-4 \lambda \tau}=0 .
$$

Multiplying $e^{2 \lambda \tau}$ on both sides of (48), we obtain

$$
A(\lambda+1)^{2}+(\lambda+1)^{4} e^{2 \lambda \tau}+C e^{-2 \lambda \tau}+B(\lambda+1) e^{-\lambda \tau}=0 .
$$

Let $\lambda=i \omega$ be a root of (49); then we have

$$
\begin{aligned}
& \bar{A}_{11} \cos 2 \tau_{1} \omega_{1}-\bar{A}_{12} \sin 2 \tau_{1} \omega_{1}+\bar{A}_{13}=\bar{A}_{14}, \\
& \bar{A}_{21} \sin 2 \tau_{1} \omega_{1}+\bar{A}_{22} \cos 2 \tau_{1} \omega_{1}+\bar{A}_{23}=\bar{A}_{24},
\end{aligned}
$$

where

$$
\begin{gathered}
\bar{A}_{11}=\omega^{4}-6 \omega^{2}+C+1, \quad \bar{A}_{12}=4 \omega-\omega^{3}, \\
\bar{A}_{13}=A-A \omega^{2}, \quad \bar{A}_{14}=B \cos \tau \omega-B \omega \sin \tau \omega, \\
\bar{A}_{21}=\omega^{4}-6 \omega^{2}-C+1, \quad \bar{A}_{22}=4 \omega-\omega^{3}, \\
\bar{A}_{23}=2 A \omega, \quad \bar{A}_{24}=B \cos \tau \omega+B \omega \sin \tau \omega .
\end{gathered}
$$

Then, we get

$$
\begin{aligned}
& \left(\bar{A}_{11} \cos 2 \tau_{1} \omega_{1}-\bar{A}_{12} \sin 2 \tau_{1} \omega_{1}+\bar{A}_{13}\right)^{2} \\
& \quad+\left(\bar{A}_{21} \sin 2 \tau_{1} \omega_{1}+\bar{A}_{22} \cos 2 \tau_{1} \omega_{1}+\bar{A}_{23}\right)^{2}=B^{2}\left(1+\omega^{2}\right) .
\end{aligned}
$$

Similar as in Case 2, we can obtain the expression of $\cos 2 \tau \omega$ and $\sin 2 \tau \omega$, which is denoted as $g_{1}(\omega)$ and $g_{2}(\omega)$, respectively. Further we can get a function with respect to $\omega$

$$
g_{1}^{2}(\omega)+g_{2}^{2}(\omega)=1
$$

Next, we make the following assumption. $\left(H_{41}\right)$ : Equation (53) has finite positive real roots, which are denoted by $\omega_{1}, \ldots, \omega_{k}$, respectively. For every fixed positive root of (53), the corresponding critical value of time delay is

$$
\begin{array}{r}
\tau_{i}^{(j)}=\frac{1}{2 \omega_{i}} \arccos g_{1}\left(\omega_{i}\right)+\frac{2 j \pi}{2 \omega_{i}}, \\
i=1, \ldots, k ; j=0,1,2, \ldots
\end{array}
$$

Then, $\pm i \omega_{i}$ are a pair of purely imaginary roots of (49) with $\tau=\tau_{i}^{(j)}$. Let

$$
\begin{gathered}
\tau_{0}=\min \left\{\tau_{i}^{(j)}\right\}, \quad \omega_{0}=\omega_{i_{0}}, \\
i=1,2, \ldots, k, j=0,1,2, \ldots .
\end{gathered}
$$

Differentiating both sides of (49) with respect to $t$, we can obtain

$$
\begin{aligned}
& {\left[\frac{d \lambda}{d \tau}\right]^{-1}} \\
& \quad=\frac{2 A(\lambda+1)+4(\lambda+1)^{3} e^{2 \lambda \tau}+B e^{-\lambda \tau}}{B \lambda(\lambda+1) e^{-\lambda \tau}+2 C \lambda e^{-2 \lambda \tau}-2 \lambda(\lambda+1)^{4} e^{2 \lambda \tau}}-\frac{\tau}{\lambda} .
\end{aligned}
$$

Thus,

$$
\operatorname{Re}\left[\frac{d \lambda}{d \tau_{2}}\right]_{\tau=\tau_{0}}^{-1}=\frac{P_{R}^{\prime} Q_{R}^{\prime}+P_{I}^{\prime} Q_{I}^{\prime}}{Q_{R}^{\prime 2}+Q_{I}^{\prime 2}},
$$


where

$$
\begin{aligned}
P_{R}^{\prime}= & \left(1-3 \omega_{0}^{2}\right) \cos 2 \tau_{0} \omega_{0}-\left(3 \omega_{0}-\omega_{0}^{3}\right) \sin 2 \tau_{0} \omega_{0} \\
& +B \cos \tau_{0} \omega_{0}+2 A \\
P_{I}^{\prime}= & \left(1-3 \omega_{0}^{2}\right) \sin 2 \tau_{0} \omega_{0}+\left(3 \omega_{0}-\omega_{0}^{3}\right) \cos 2 \tau_{0} \omega_{0} \\
& -B \cos \tau_{0} \omega_{0}+2 A \omega_{0}, \\
Q_{R}^{\prime}= & B \omega_{0} \sin \tau_{0} \omega_{0}-B \omega_{0}^{2} \cos \tau_{0} \omega_{0} \\
& +\left(\omega_{0}^{5}+(1+2 C) \omega_{0}-6 \omega_{0}^{6}\right) \sin 2 \tau_{0} \omega_{0} \\
& -8\left(\omega_{0}^{4}-\omega_{0}^{2}\right) \cos 2 \tau_{0} \omega_{0}, \\
Q_{I}^{\prime}= & B \omega_{0} \cos \tau_{0} \omega_{0}+B \omega_{0}^{2} \sin \tau_{0} \omega_{0} \\
& -\left(\omega_{0}^{5}+(1+2 C) \omega_{0}-6 \omega_{0}^{6}\right) \cos 2 \tau_{0} \omega_{0} \\
& -8\left(\omega_{0}^{4}-\omega_{0}^{2}\right) \sin 2 \tau_{0} \omega_{0} .
\end{aligned}
$$

Obviously, if the condition $\left(H_{42}\right): P_{R}^{\prime} Q_{R}^{\prime}+P_{I}^{\prime} Q_{I}^{\prime} \neq 0$ holds, then $\operatorname{Re}\left[d \lambda / d \tau_{2}\right]_{\tau=\tau_{0}}^{-1} \neq 0$. Namely, if the condition $\left(H_{42}\right)$ holds, the transversality condition is satisfied. Thus, by the Hopf bifurcation theorem in [23] we have the following results.

Theorem 3. If the condition $\left(H_{41}\right)$ means that (53) has finite positive real roots and $\left(H_{42}\right)$ means that $P_{R}^{\prime} Q_{R}^{\prime}+P_{I}^{\prime} Q_{I}^{\prime} \neq 0$ holds, then the zero equilibrium $E_{0}$ of system (3) is asymptotically stable for $\tau \in\left[0, \tau_{0}\right)$, system (3) undergoes a Hopf bifurcation at $E_{0}$ when $\tau=\tau_{0}$, and a branch of periodic solutions bifurcates from the zero equilibrium near $\tau=\tau_{0}$.

Case $5\left(\tau_{1}>0\right.$ and $\left.\tau_{2}>0\right)$. We consider (5) with $\tau_{1}$ in its stable interval and $\tau_{2}$ is considered as a parameter. Without loss of generality, we consider (5) under Case 2.

Let $\lambda=i \omega_{2 *}\left(\omega_{2 *}>0\right)$ be a root of (5). Then, we can get

$$
\begin{aligned}
\omega^{8} & +4 \omega^{6}+6 \omega^{4}+4 \omega^{2}+1+2 B\left(A \omega^{3}+C \omega\right) \sin \tau_{1} \omega \\
& -2 B(A+C) \cos \tau_{1} \omega+2 A C\left(\omega^{2}-1\right) \cos 2 \tau_{1} \omega \\
& +2 A C \omega \sin 2 \tau_{1} \omega=0 .
\end{aligned}
$$

Suppose that $\left(H_{51}\right)$ means that (59) has finite positive real roots, which are denoted as $\omega_{21 *}, \omega_{22 *}, \ldots, \omega_{2 k *}$. For every positive real root $\omega_{2 i *}(i=1,2, \ldots, k)$, their exists a sequence $\left\{\tau_{2 i *}^{(j)} \mid j=0,1,2, \ldots\right\}$, such that (59) has a pair of purely imaginary roots $\pm i \omega_{2 i *}$ when $\tau_{2}=\tau_{2 i *}^{(j)}$.

Let $\tau_{2}^{*}=\min \left\{\tau_{2 i *}^{(j)} \mid j=0,1,2, \ldots\right\}$, and when $\tau_{2}=\tau_{2}^{*}(59)$ has a pair of purely imaginary roots $\pm i \omega_{2}^{*}$. In the following, we make the following assumption.

$$
\left(H_{52}\right): \operatorname{Re}\left[d \lambda / d \tau_{2}\right]_{\tau_{2}=\tau_{2}^{*}}^{-1} \neq 0 .
$$

Through the analysis above and by the Hopf bifurcation theorem in [23], we have the following results.
Theorem 4. If the condition $\left(H_{51}\right)$ means that (59) has finite positive real roots and $\left(H_{52}\right)$ means that $\operatorname{Re}\left[d \lambda / d \tau_{2}\right]_{\tau_{2}=\tau_{2}^{*}}^{-1} \neq 0$ holds, and $\tau_{1} \in\left(0, \tau_{10}\right)$, then the zero equilibrium $E_{0}$ of system (3) is asymptotically stable for $\tau_{2} \in\left[0, \tau_{2}^{*}\right)$, system (3) undergoes a Hopf bifurcation at $E_{0}$ when $\tau_{2}=\tau_{2}^{*}$, and a branch of periodic solutions bifurcates from the zero equilibrium near $\tau_{2}=\tau_{2}^{*}$.

\section{Stability of Bifurcated Periodic Solutions}

In this section, the formulae for determining the direction of Hopf bifurcation and the stability of bifurcating periodic solutions of system (3) with respect to $\tau_{2}$ for $\tau_{1} \in\left(0, \tau_{10}\right)$ are derived by using the normal form method and center manifold theorem introduced by Hassard et al. [23]. Throughout this section, it is considered that system (3) undergoes Hopf bifurcation at $\tau_{2}=\tau_{2}^{*}$ and $\tau_{1} \in\left(0, \tau_{10}\right)$. Without loss of generality, we assume that $\tau_{1}^{*}<\tau_{2}^{*}$, where $\tau_{1}^{*} \in\left(0, \tau_{10}\right)$.

For convenience, let $t=s \tau_{2}, \bar{u}_{i}(t)=u_{i}\left(\tau_{2} t\right),(i=$ $1,2,3,4)$. Drop the bars for simplification of notations. Then system (3) becomes

$$
\dot{u}(t)=L_{\mu} u_{t}+F\left(\mu, u_{t}\right),
$$

where $u(t)=\left(u_{1}(t), u_{2}(t), u_{3}(t), u_{4}(t)\right)^{T} \in C=C\left([-1,0], R^{4}\right)$ and $L_{\mu}: C \rightarrow R^{4}, F: R \times C \rightarrow R^{4}$ are given, respectively, by

$$
\begin{gathered}
L_{\mu} \phi=\left(\tau_{2}^{*}+\mu\right)\left(A^{\prime} \phi(0)+B^{\prime} \phi\left(-\frac{\tau_{1}^{*}}{\tau_{2}^{*}}\right)+C^{\prime} \phi(-1)\right), \\
F(\mu, \phi)=\left(\tau_{2}^{*}+\mu\right)\left(F_{1}, F_{2}, F_{3}, F_{4}\right)^{T},
\end{gathered}
$$

with

$$
\begin{aligned}
\phi(\theta) & =\left(\phi_{1}(\theta), \phi_{2}(\theta), \phi_{3}(\theta), \phi_{4}(\theta)\right)^{T} \in C\left([-1,0], R^{4}\right), \\
A^{\prime} & =\left(\begin{array}{cccc}
-1 & 0 & 0 & 0 \\
0 & -1 & 0 & 0 \\
0 & 0 & -1 & 0 \\
0 & 0 & 0 & -1
\end{array}\right), \\
B^{\prime} & =\left(\begin{array}{cccc}
0 & f^{\prime}(0) & 0 & 0 \\
0 & 0 & f^{\prime}(0) & 0 \\
0 & 0 & 0 & f^{\prime}(0) \\
0 & 0 & 0 & 0
\end{array}\right), \\
C^{\prime} & =\left(\begin{array}{cccc}
0 & 0 & 0 & 0 \\
0 & 0 & 0 & 0 \\
0 & 0 & 0 & 0 \\
w_{1} f^{\prime}(0) & w_{2} f^{\prime}(0) & w_{3} f^{\prime}(0) & 0
\end{array}\right), \\
F_{1} & =\frac{f^{\prime \prime}}{2 !} \phi_{2}^{2}\left(-\frac{\tau_{1}^{*}}{\tau_{2}^{*}}\right)+\frac{f^{\prime \prime \prime}}{3 !} \phi_{2}^{3}\left(-\frac{\tau_{1}^{*}}{\tau_{2}^{*}}\right)+\cdots,
\end{aligned}
$$




$$
\begin{aligned}
F_{2}= & \frac{f^{\prime \prime}}{2 !} \phi_{3}^{2}\left(-\frac{\tau_{1}^{*}}{\tau_{2}^{*}}\right)+\frac{f^{\prime \prime \prime}}{3 !} \phi_{3}^{3}\left(-\frac{\tau_{1}^{*}}{\tau_{2}^{*}}\right)+\cdots, \\
F_{3}= & \frac{f^{\prime \prime}}{2 !} \phi_{4}^{2}\left(-\frac{\tau_{1}^{*}}{\tau_{2}^{*}}\right)+\frac{f^{\prime \prime \prime}}{3 !} \phi_{4}^{3}\left(-\frac{\tau_{1}^{*}}{\tau_{2}^{*}}\right)+\cdots, \\
F_{4}= & \frac{w_{1} f^{\prime \prime}(0)}{2 !} \phi_{1}^{2}(-1)+\frac{w_{1} f^{\prime \prime \prime}(0)}{3 !} \phi_{1}^{3}(-1) \\
& +\frac{w_{2} f^{\prime \prime}(0)}{2 !} \phi_{2}^{2}(-1)+\frac{w_{2} f^{\prime \prime \prime}(0)}{3 !} \phi_{2}^{3}(-1) \\
& +\frac{w_{3} f^{\prime \prime}(0)}{2 !} \phi_{1}^{2}(-1)+\frac{w_{3} f^{\prime \prime \prime}(0)}{3 !} \phi_{3}^{3}(-1)+\cdots
\end{aligned}
$$

Therefore, according to the Riesz representation theorem, there exists a $4 \times 4$ matrix function $\eta(\theta, \mu):[-1,0] \rightarrow R^{4}$ whose elements are of bounded variation such that

$$
L_{\mu} \phi=\int_{-1}^{0} d \eta(\theta, \mu) \phi(\theta), \quad \phi \in C\left([-1,0], R^{4}\right) .
$$

In fact, we choose

$$
\begin{aligned}
\eta(\theta, \mu) & \begin{cases}\left(\tau_{2}^{*}+\mu\right)\left(A^{\prime}+B^{\prime}+C^{\prime}\right), & \theta=0, \\
\left(\tau_{2}^{*}+\mu\right)\left(B^{\prime}+C^{\prime}\right), & \theta \in\left[-\frac{\tau_{1}^{*}}{\tau_{2}^{*}}, 0\right), \\
\left(\tau_{2}^{*}+\mu\right) C^{\prime}, & \theta \in\left(-1,-\frac{\tau_{1}^{*}}{\tau_{2}^{*}}\right), \\
0, & \theta=-1 .\end{cases}
\end{aligned}
$$

For $\phi \in C\left([-1,0], R^{4}\right)$, we define

$$
\begin{gathered}
A(\mu) \phi= \begin{cases}\frac{d \phi(\theta)}{d \theta}, & -1 \leq \theta<0, \\
\int_{-1}^{0} d \eta(\theta, \mu) \phi(\theta), & \theta=0,\end{cases} \\
R(\mu) \phi= \begin{cases}0, & -1 \leq \theta<0, \\
F(\mu, \phi), & \theta=0 .\end{cases}
\end{gathered}
$$

Then system (60) can be transformed into the following operator equation:

$$
\dot{u}(t)=A(\mu) u_{t}+R(\mu) u_{t},
$$

where $u_{t}=u(t+\theta)$ for $\theta \in[-1,0]$.

For $\varphi \in C^{\prime}\left([0,1],\left(R^{4}\right)^{*}\right)$, where $\left(R^{4}\right)^{*}$ is the 4 dimensional space of row vector, we define the adjoint operator $A^{*}$ of $A$ :

$$
A^{*}(\varphi)= \begin{cases}-\frac{d \varphi(s)}{d s}, & 0<s \leq 1, \\ \int_{-1}^{0} d \eta^{T}(s, 0) \varphi(-s), & s=0,\end{cases}
$$

and a bilinear inner product

$$
\begin{aligned}
& \langle\varphi(s), \phi(\theta)\rangle \\
& =\bar{\varphi}(0) \phi(0)-\int_{\theta=-1}^{0} \int_{\xi=0}^{\theta} \bar{\varphi}(\xi-\theta) d \eta(\theta) \phi(\xi) d \xi,
\end{aligned}
$$

where $\eta(\theta)=\eta(\theta, 0)$.

Then $A(0)$ and $A^{*}(0)$ are adjoint operators. From the discussion above, we know that $\pm i \omega_{2}^{*} \tau_{2}^{*}$ are eigenvalues of $A(0)$ and they are also eigenvalues of $A^{*}(0)$. Let $q(\theta)=\left(1, q_{2}, q_{3}, q_{4}\right)^{T} e^{i \omega_{2}^{*} \tau_{2}^{*} \theta}$ be the eigenvector of $A(0)$ corresponding to the eigenvalue $+i \omega_{2}^{*} \tau_{2}^{*}$, and let $q^{*}(s)=$ $D\left(1, q_{2}^{*}, q_{3}^{*}, q_{4}^{*}\right) e^{i \omega_{2}^{*} \tau_{2}^{*} s}$ be the eigenvector of $A^{*}(0)$ corresponding to the eigenvalue $-i \omega_{2}^{*} \tau_{2}^{*}$. Then, we have

$$
A(0) q(\theta)=i \omega_{2}^{*} \tau_{2}^{*} q(\theta), \quad A^{*}(0) q^{*}(0)=-i \omega_{2}^{*} \tau_{2}^{*} q^{*}(\theta) .
$$

By a simple computation, we can obtain

$$
\begin{aligned}
& q_{2}=\frac{i \omega_{2}^{*}+1}{f^{\prime}(0) e^{-i \omega_{2}^{*} \tau_{1}^{*}}}, \quad q_{3}=\frac{q_{2}}{f^{\prime}(0) e^{-i \omega_{2}^{*} \tau_{1}^{*}-i \omega_{2}^{*}},} \\
& q_{4}=\frac{f^{\prime}(0)\left(w_{1}+w_{2} q_{2}+w_{3} q_{3}\right)}{\left(i \omega_{2}^{*}+1\right) e^{i \omega_{2}^{*} \tau_{2}^{*}}}, \\
& q_{4}^{*}=\frac{1-i \omega_{2}^{*}}{w_{1} f^{\prime}(0) e^{i \omega_{2}^{*} \tau_{2}^{*}}}, \quad q_{2}^{*}=\frac{e^{i \omega_{2}^{*} \tau_{1}^{*}}+w_{2} q_{4}^{*} e^{i \omega_{2}^{*} \tau_{2}^{*}}}{1-i \omega_{2}^{*}} f^{\prime}(0), \\
& q_{3}^{*}=\frac{q_{2}^{*} e^{i \omega_{2}^{*} \tau_{1}^{*}}+w_{3} q_{4}^{*} e^{i \omega_{2}^{*} \tau_{2}^{*}}}{1-i \omega_{2}^{*}} f^{\prime}(0)
\end{aligned}
$$

and $\left\langle q^{*}, q\right\rangle=1,\left\langle q^{*}, \bar{q}\right\rangle=0$.

From (68), we can get

$$
\begin{aligned}
\bar{D}=[ & 1+q_{2} \bar{q}_{2}^{*}+q_{3} \bar{q}_{3}^{*}+q_{4} \bar{q}_{4}^{*} \\
& +\tau_{1}^{*} f^{\prime}(0)\left(q_{2}+\bar{q}_{2}^{*} q_{3}+\bar{q}_{3}^{*} q_{4}\right) e^{-i \omega_{2}^{*} \tau_{1}^{*}} \\
& \left.+\tau_{2}^{*} f^{\prime}(0) \bar{q}_{4}^{*}\left(w_{1}+w_{2} q_{2}+w_{3} q_{3}\right) e^{-i \omega_{2}^{*} \tau_{2}^{*}}\right]^{-1} .
\end{aligned}
$$

Following the algorithms given in [23] and using similar computation process in [24], we can get the coefficients which can be used to determine direction of the Hopf bifurcation and stability of the bifurcating periodic solutions:

$$
\begin{gathered}
g_{20}=f^{\prime \prime}(0) \bar{D}\left[e^{-2 i \omega_{2}^{*} \tau_{1}^{*}}\left(q_{2}^{2}+\bar{q}_{2}^{*} q_{3}^{2}+\bar{q}_{3}^{*} q_{4}^{2}\right)\right. \\
\left.+\bar{q}_{4}^{*} e^{-2 i \omega_{2}^{*} \tau_{2}^{*}}\left(w_{1}+w_{2} q_{2}^{2}+w_{3} q_{3}^{2}\right)\right], \\
g_{11}=f^{\prime \prime}(0) \bar{D}\left[q_{2} \bar{q}_{2}+\bar{q}_{2}^{*} q_{3} \bar{q}_{3}+\bar{q}_{3}^{*} q_{4} \bar{q}_{4}\right. \\
\left.+\bar{q}_{4}^{*}\left(w_{1}+w_{2} q_{2} \bar{q}_{2}+w_{3} q_{3} \bar{q}_{3}\right)\right],
\end{gathered}
$$




$$
\begin{aligned}
& g_{02}=f^{\prime \prime}(0) \bar{D}\left[e^{2 i \omega_{2}^{*} \tau_{1}^{*}}\left(\bar{q}_{2}^{2}+\bar{q}_{2}^{*} \bar{q}_{3}^{2}+\bar{q}_{3}^{*} \bar{q}_{4}^{2}\right)\right. \\
& \left.+\bar{q}_{4}^{*} e^{2 i \omega_{2}^{*} \tau_{2}^{*}}\left(w_{1}+w_{2} \bar{q}_{2}^{2}+w_{3} \bar{q}_{3}^{2}\right)\right], \\
& g_{21}=\bar{D}\left[f ^ { \prime \prime } ( 0 ) \left(2 W_{11}^{(2)}\left(-\frac{\tau_{1}^{*}}{\tau_{2}^{*}}\right) q_{2} e^{-i \omega_{2}^{*} \tau_{1}^{*}}\right.\right. \\
& \left.+W_{20}^{(2)}\left(-\frac{\tau_{1}^{*}}{\tau_{2}^{*}}\right) \bar{q}_{2} e^{i \omega_{2}^{*} \tau_{1}^{*}}\right) \\
& +f^{\prime \prime \prime}(0) q_{2}^{2} \bar{q}_{2} e^{-i \omega_{2}^{*} \tau_{1}^{*}} \\
& +\bar{q}_{2}^{*}\left(f ^ { \prime \prime } ( 0 ) \left(2 W_{11}^{(3)}\left(-\frac{\tau_{1}^{*}}{\tau_{2}^{*}}\right) q_{3} e^{-i \omega_{2}^{*} \tau_{1}^{*}}\right.\right. \\
& \left.+W_{20}^{(3)}\left(-\frac{\tau_{1}^{*}}{\tau_{2}^{*}}\right) \bar{q}_{3} e^{i \omega_{2}^{*} \tau_{1}^{*}}\right) \\
& \left.+f^{\prime \prime \prime}(0) q_{3}^{2} \bar{q}_{3} e^{-i \omega_{2}^{*} \tau_{1}^{*}}\right) \\
& +\bar{q}_{3}^{*}\left(f ^ { \prime \prime } ( 0 ) \left(2 W_{11}^{(4)}\left(-\frac{\tau_{1}^{*}}{\tau_{2}^{*}}\right) q_{2} e^{-i \omega_{2}^{*} \tau_{1}^{*}}\right.\right. \\
& \left.+W_{20}^{(4)}\left(-\frac{\tau_{1}^{*}}{\tau_{2}^{*}}\right) \bar{q}_{2} e^{i \omega_{2}^{*} \tau_{1}^{*}}\right) \\
& \left.+f^{\prime \prime \prime}(0) q_{4}^{2} \bar{q}_{4} e^{-i \omega_{2}^{*} \tau_{1}^{*}}\right) \\
& +\bar{q}_{4}^{*}\left(w _ { 1 } f ^ { \prime \prime } ( 0 ) \left(2 W_{11}^{(1)}(-1) e^{-i \omega_{2}^{*} \tau_{2}^{*}}\right.\right. \\
& \left.+W_{20}^{(1)}(-1) e^{i \omega_{2}^{*} \tau_{2}^{*}}\right) \\
& +w_{1} f^{\prime \prime \prime}(0) e^{-i \omega_{2}^{*} \tau_{2}^{*}} \\
& +w_{2} f^{\prime \prime}(0)\left(2 W_{11}^{(2)}(-1) q_{2} e^{-i \omega_{2}^{*} \tau_{2}^{*}}\right. \\
& \left.+W_{20}^{(2)}(-1) \bar{q}_{2} e^{i \omega_{2}^{*} \tau_{2}^{*}}\right) \\
& +w_{2} f^{\prime \prime \prime}(0) q_{2}^{2} \bar{q}_{2} e^{-i \omega_{2}^{*} \tau_{2}^{*}} \\
& +w_{3} f^{\prime \prime}(0)\left(2 W_{11}^{(3)}(-1) q_{3} e^{-i \omega_{2}^{*} \tau_{2}^{*}}\right. \\
& \left.+W_{20}^{(3)}(-1) \bar{q}_{3} e^{i \omega_{2}^{*} \tau_{2}^{*}}\right) \\
& \left.\left.+w_{3} f^{\prime \prime \prime}(0) q_{3}^{2} \bar{q}_{3} e^{-i \omega_{2}^{*} \tau_{2}^{*}}\right)\right],
\end{aligned}
$$

with

$$
\begin{gathered}
W_{20}(\theta)=\frac{i g_{20} q(0)}{\omega_{2}^{*} \tau_{2}^{*}} e^{i \omega_{2}^{*} \tau_{2}^{*} \theta}+\frac{i \bar{g}_{02} \bar{q}(0)}{3 \omega_{2}^{*} \tau_{2}^{*}} e^{-i \omega_{2}^{*} \tau_{2}^{*} \theta}+E_{1} e^{2 i \omega_{2}^{*} \tau_{2}^{*} \theta}, \\
W_{11}(\theta)=-\frac{i g_{11} q(0)}{\omega_{2}^{*} \tau_{2}^{*}} e^{i \omega_{2}^{*} \tau_{2}^{*} \theta}+\frac{i \bar{g}_{11} \bar{q}(0)}{\omega_{2}^{*} \tau_{2}^{*}} e^{-i \omega_{2}^{*} \tau_{2}^{*} \theta}+E_{2},
\end{gathered}
$$

where $E_{1}$ and $E_{2}$ can be computed by the following equations, respectively:

$$
\begin{aligned}
& \left(\begin{array}{cccc}
2 i \omega_{2}^{*}+1 & \alpha_{12} & 0 & 0 \\
0 & 2 i \omega_{2}^{*}+1 & \alpha_{23} & 0 \\
0 & 0 & 2 i \omega_{2}^{*}+1 & \alpha_{34} \\
\alpha_{41} & \alpha_{42} & \alpha_{43} & 2 i \omega_{2}^{*}+1
\end{array}\right) E_{1} \\
& =\left(\begin{array}{c}
E_{1}^{(1)} \\
E_{1}^{(2)} \\
E_{1}^{(3)} \\
E_{1}^{(4)}
\end{array}\right),
\end{aligned}
$$$$
\left(\begin{array}{cccc}
1 & -f^{\prime}(0) & 0 & 0 \\
0 & 1 & -f^{\prime}(0) & 0 \\
0 & 0 & 1 & -f^{\prime}(0) \\
-w_{1} f^{\prime}(0) & -w_{2} f^{\prime}(0) & -w_{3} f^{\prime}(0) & 1
\end{array}\right) E_{2}
$$$$
=-\left(\begin{array}{c}
E_{2}^{(1)} \\
E_{2}^{(2)} \\
E_{2}^{(3)} \\
E_{2}^{(4)}
\end{array}\right) \text {, }
$$

with

$$
\begin{aligned}
& \alpha_{12}=\alpha_{23}=\alpha_{34}=-f^{\prime}(0) e^{-2 i \omega_{2}^{*} \tau_{1}^{*}}, \\
& \alpha_{41}=-w_{1} f^{\prime}(0) e^{-2 i \omega_{2}^{*} \tau_{2}^{*}}, \\
& \alpha_{42}=-w_{2} f^{\prime}(0) e^{-2 i \omega_{2}^{*} \tau_{2}^{*}}, \\
& \alpha_{43}=-w_{3} f^{\prime}(0) e^{-2 i \omega_{2}^{*} \tau_{2}^{*}} . \\
& E_{1}^{(1)}=f^{\prime \prime}(0) q_{2}^{2} e^{-2 i \omega_{2}^{*} \tau_{1}^{*}}, \\
& E_{1}^{(2)}=f^{\prime \prime}(0) q_{3}^{2} e^{-2 i \omega_{2}^{*} \tau_{1}^{*}}, \\
& E_{1}^{(3)}=f^{\prime \prime}(0) q_{4}^{2} e^{-2 i \omega_{2}^{*} \tau_{1}^{*}}, \\
& E_{1}^{(4)}=f^{\prime \prime}(0)\left(w_{1}+w_{2} q_{2}^{2}+w_{3} q_{3}^{2}\right) e^{-2 i \omega_{2}^{*} \tau_{2}^{*}}, \\
& E_{2}^{(1)}=f^{\prime \prime}(0) q_{2} \bar{q}_{2}, \\
& E_{2}^{(2)}=f^{\prime \prime}(0) q_{3} \bar{q}_{3}, \\
& E_{2}^{(3)}=f^{\prime \prime}(0) q_{4} \bar{q}_{4}, \\
& E_{2}^{(4)}=f^{\prime \prime}(0)\left(w_{1}+w_{2} q_{2} \bar{q}_{2}+w_{3} q_{3} \bar{q}_{3}\right) .
\end{aligned}
$$




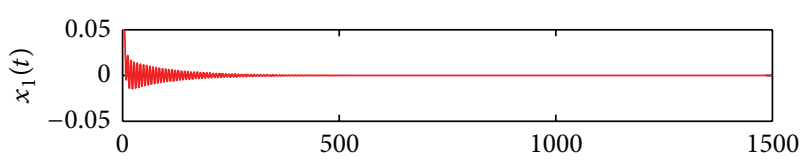

(a)

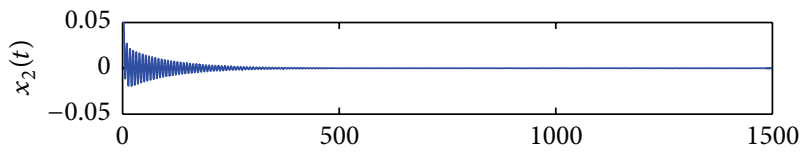

(b)

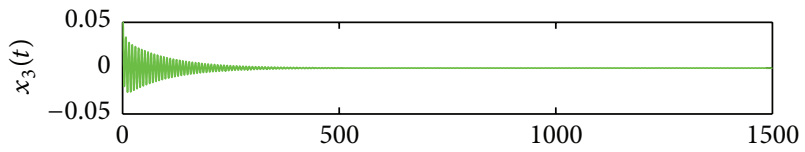

(c)

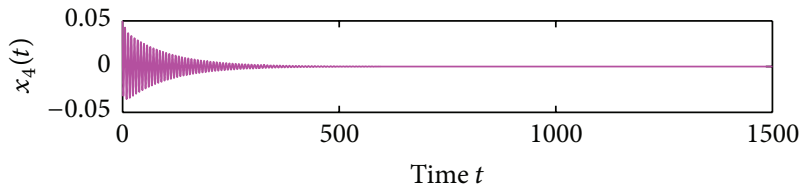

(d)

FIgURE 1: The trajectory of $x_{1}, x_{2}, x_{3}$, and $x_{4}$ when $\tau_{1}=1.3<$ $1.4022=\tau_{10}$.

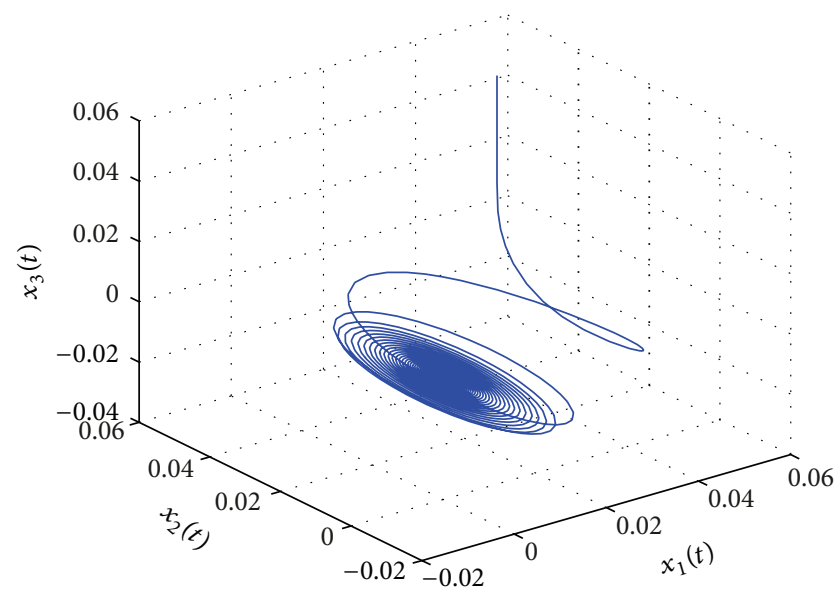

FIGURE 2: The phase plot of $x_{1}, x_{2}$, and $x_{3}$ when $\tau_{1}=1.3<1.4022=$ $\tau_{10}$.

Therefore, we can calculate the following values:

$$
\begin{gathered}
C_{1}(0)=\frac{i}{2 \omega_{2}^{*} \tau_{2}^{*}}\left(g_{11} g_{20}-2\left|g_{11}\right|^{2}-\frac{\left|g_{02}\right|^{2}}{3}\right)+\frac{g_{21}}{2}, \\
\mu_{2}=-\frac{\operatorname{Re}\left\{C_{1}(0)\right\}}{\operatorname{Re}\left\{\lambda^{\prime}\left(\tau_{2}^{*}\right)\right\}}, \\
\beta_{2}=2 \operatorname{Re}\left\{C_{1}(0)\right\} \\
T_{2}=-\frac{\operatorname{Im}\left\{C_{1}(0)\right\}+\mu_{2} \operatorname{Im}\left\{\lambda^{\prime}\left(\tau_{2}^{*}\right)\right\}}{\omega_{2}^{*} \tau_{2}^{*}} .
\end{gathered}
$$

Based on the discussion above, we can obtain the following results.

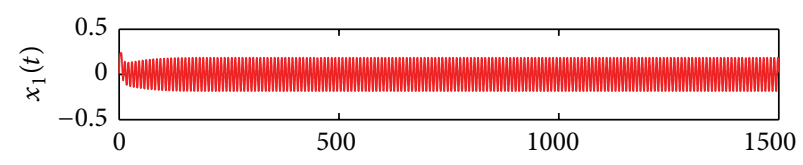

(a)

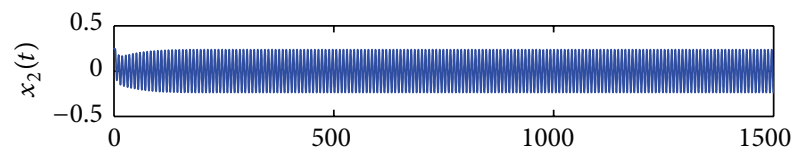

(b)

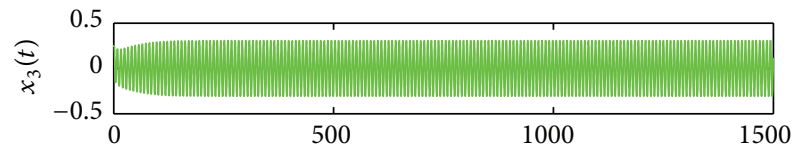

(c)

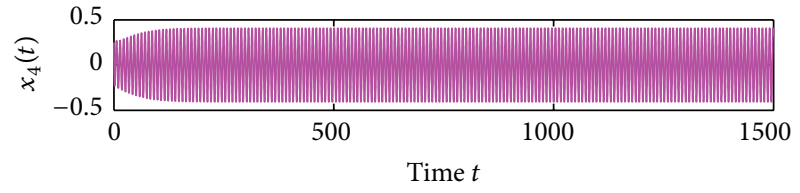

(d)

FIGURE 3: The trajectory of $x_{1}, x_{2}, x_{3}$, and $x_{4}$ when $\tau_{1}=1.5>$ $1.4022=\tau_{10}$.

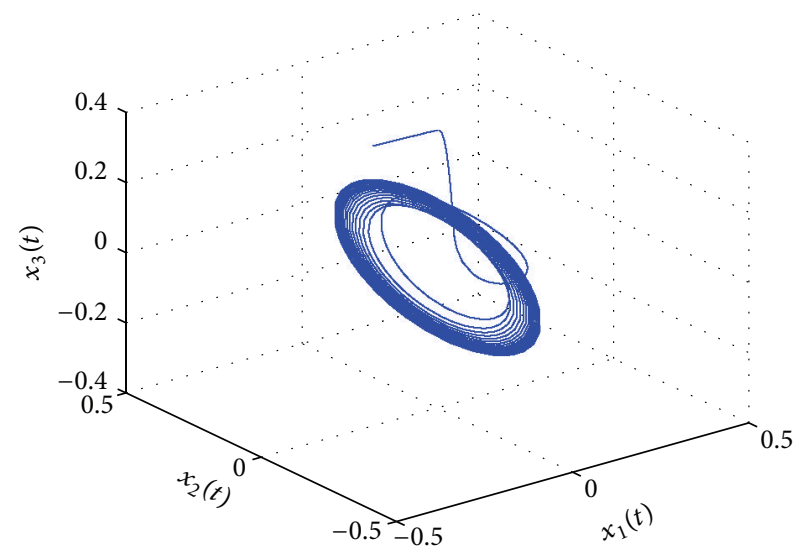

FIGURE 4: The phase plot of $x_{1}, x_{2}$, and $x_{3}$ when $\tau_{1}=1.5>1.4022=$ $\tau_{10}$.

Theorem 5. For system (3),

(i) $\mu_{2}$ determines the direction of the Hopf bifurcation. If $\mu_{2}>0\left(\mu_{2}<0\right)$; then the Hopf bifurcation is supercritical (subcritical);

(ii) $\beta_{2}$ determines the stability of the bifurcating periodic solutions. If $\beta_{2}<0\left(\beta_{2}>0\right)$; then the bifurcating periodic solutions are stable (unstable);

(iii) $T_{2}$ determines the period of the bifurcating periodic solutions. If $T_{2}>0\left(T_{2}<0\right)$; then the period of the bifurcating periodic solutions increases (decreases). 


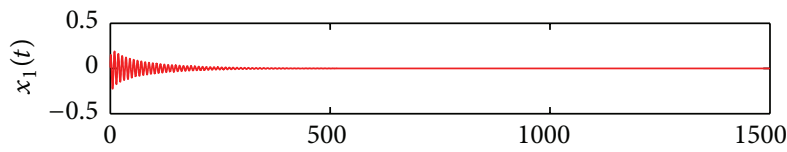

(a)

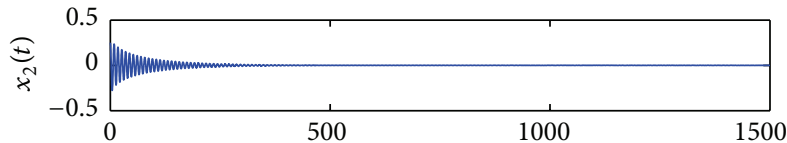

(b)

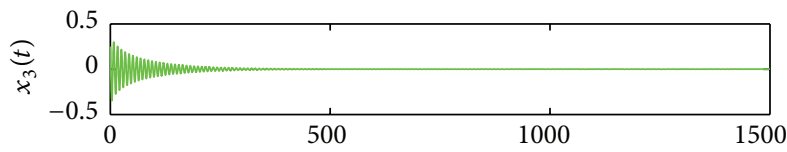

(c)

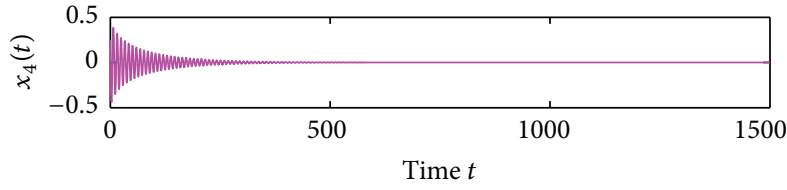

(d)

FIGURE 5: The trajectory of $x_{1}, x_{2}, x_{3}$, and $x_{4}$ when $\tau_{2}=2.75<$ $3.1610=\tau_{20}$.

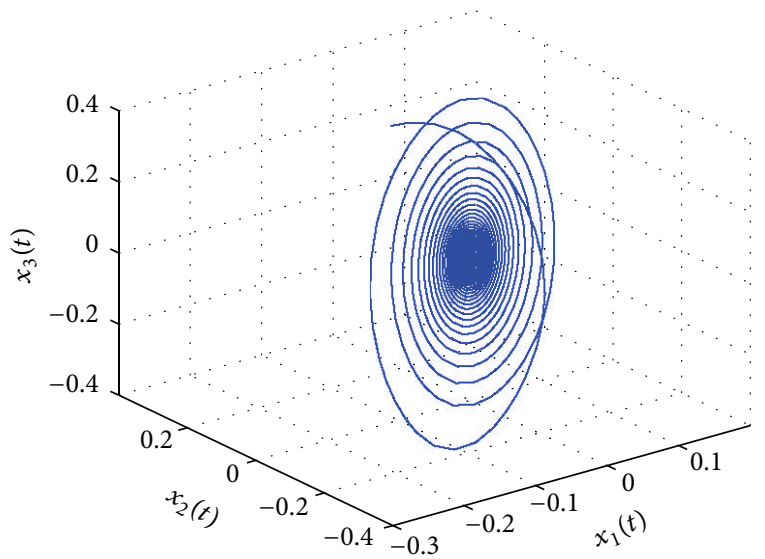

FIGURE 6: The phase plot of $x_{1}, x_{2}$, and $x_{3}$ when $\tau_{2}=2.75<3.1610=$ $\tau_{20}$.

\section{Numerical Simulation}

In this section, we present some numerical simulations to support the theoretical analysis in Sections 2 and 3. As an example, we consider the following special case of system (3) with the parameters $w_{1}=1, w_{2}=-1, w_{3}=-1$, and $f(x)=\tanh (x)$. Then $f(0)=0, f^{\prime}(0)=1$, and system $(3)$ becomes

$$
\begin{aligned}
& \dot{x}_{1}(t)=-x_{1}(t)+\tanh \left(x_{2}\left(t-\tau_{1}\right)\right), \\
& \dot{x}_{2}(t)=-x_{2}(t)+\tanh \left(x_{3}\left(t-\tau_{1}\right)\right), \\
& \dot{x}_{3}(t)=-x_{3}(t)+\tanh \left(x_{4}\left(t-\tau_{1}\right)\right),
\end{aligned}
$$

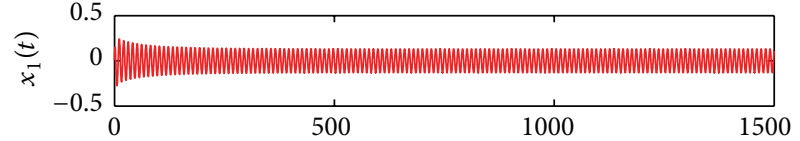

(a)

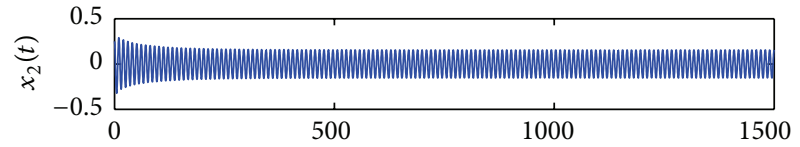

(b)

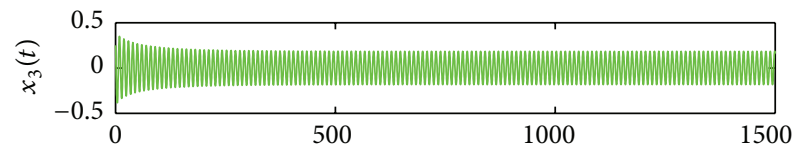

(c)

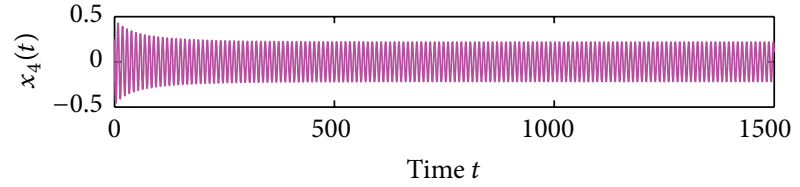

(d)

FIGURE 7: The trajectory of $x_{1}, x_{2}, x_{3}$, and $x_{4}$ when $\tau_{2}=3.5>$ $3.1610=\tau_{20}$.

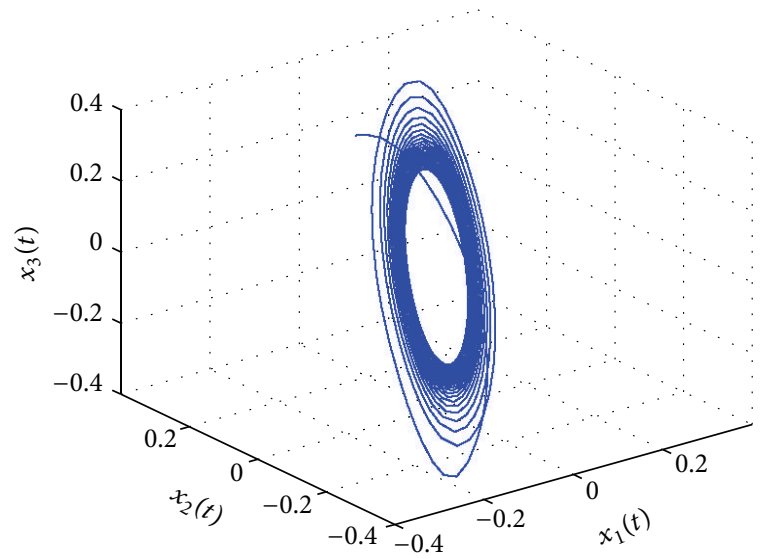

FIGURE 8: The phase plot of $x_{1}, x_{2}$, and $x_{3}$ when $\tau_{2}=3.5>3.1610=$ $\tau_{20}$.

$$
\begin{aligned}
\dot{x}_{4}(t)= & -x_{4}(t)+\tanh \left(x_{1}\left(t-\tau_{2}\right)\right) \\
& -\tanh \left(x_{2}\left(t-\tau_{2}\right)\right)-\tanh \left(x_{3}\left(t-\tau_{2}\right)\right) .
\end{aligned}
$$

Obviously, $E_{0}(0,0,0,0)$ is the equilibrium of system (77). By a simple computation, we get $D_{2}=21>0, D_{3}=131>0$, and $D_{4}=131>0$. That is, the condition $\left(H_{1}\right)$ holds.

For $\tau_{1}>0, \tau_{2}=0$. We can obtain $\omega_{10}=1.7216, \tau_{10}=$ 1.4022 by some complicated computations. From Theorem 1 , we know that $E_{0}(0,0,0,0)$ is asymptotically stable when $\tau_{1}<$ $\tau_{10}$ as illustrated by Figures 1 and 2 . When $\tau_{1}$ passes through, the critical value $\tau_{10}, E_{0}(0,0,0,0)$ becomes unstable and a 


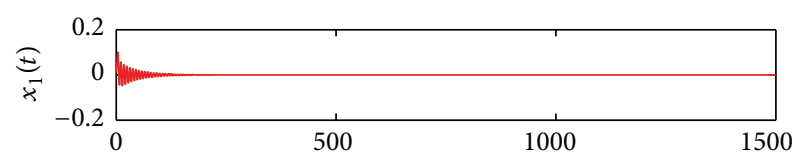

(a)

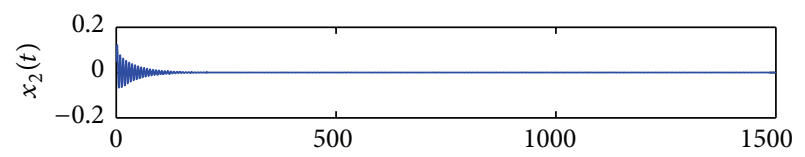

(b)

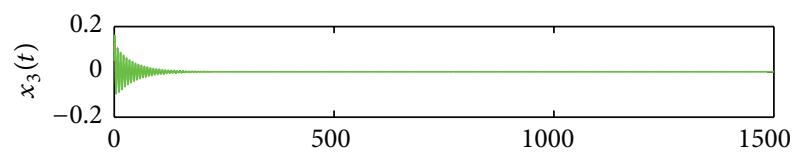

(c)

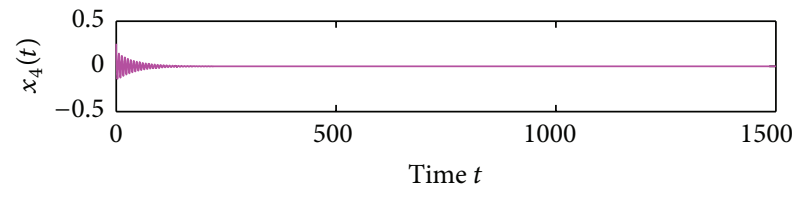

(d)

FIgURE 9: The trajectory of $x_{1}, x_{2}, x_{3}$, and $x_{4}$ when $\tau=0.7<$ $0.7915=\tau_{0}$.

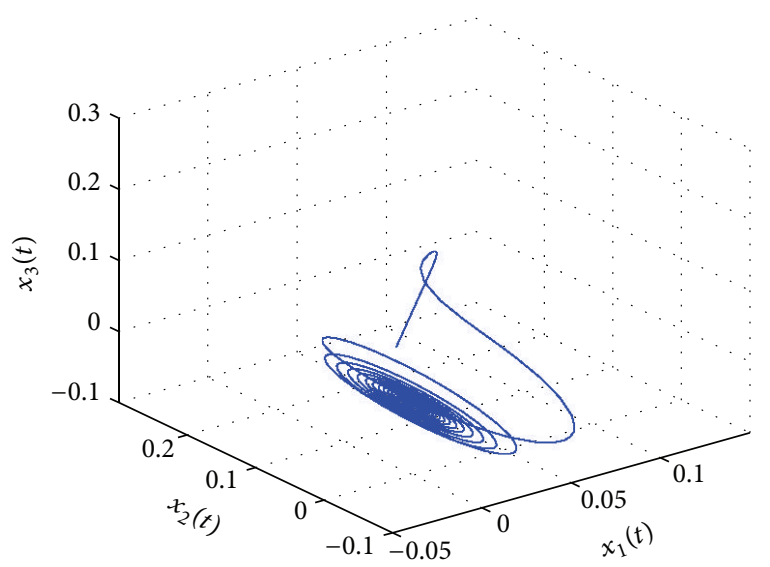

FIGURE 10: The phase plot of $x_{1}, x_{2}$, and $x_{3}$ when $\tau=0.7<0.7915=$ $\tau_{0}$.

Hopf bifurcation occurs and a branch of periodic solutions bifurcate from $E_{0}(0,0,0,0)$, which can be seen from Figures 3 and 4. Similarly, we have $\omega_{20}=0.5194, \tau_{20}=3.1610$ for $\tau_{1}=0, \tau_{2}>0$. The corresponding waveforms and the phase plots are shown in Figures 5, 6, 7, and 8 .

For $\tau_{1}=\tau_{2}=\tau>0$, we obtain $\omega_{0}=2.0967, \tau_{0}=0.7915$. From Theorem 3, when $\tau$ increases from zero to the critical value $\tau_{0}, E_{0}(0,0,0,0)$ is asymptotically stable, then it will lose its stability and a Hopf bifurcation occurs once $\tau>\tau_{0}$. These properties can be shown in Figures 9, 10, 11, and 12 .

Lastly, for $\tau_{2}>0$ and $\tau_{1}^{*}=0.35 \in\left(0, \tau_{10}\right)$, we get $\omega_{2}^{*}=1.3743, \tau_{2}^{*}=1.7488$. By Theorem $4, E_{0}(0,0,0,0)$ is asymptotically stable when $\tau_{2} \in\left[0, \tau_{2}^{*}\right)$, and $E_{0}(0,0,0,0)$ is

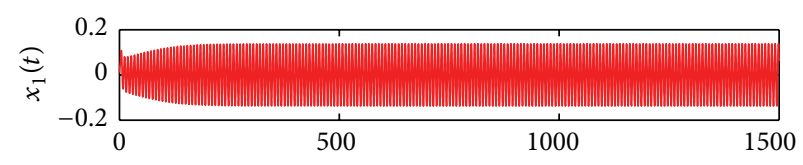

(a)

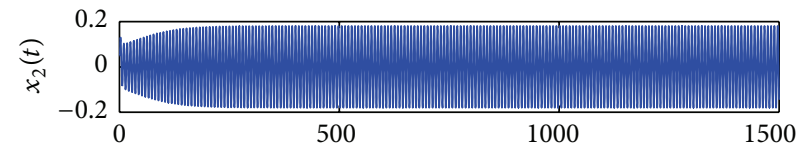

(b)

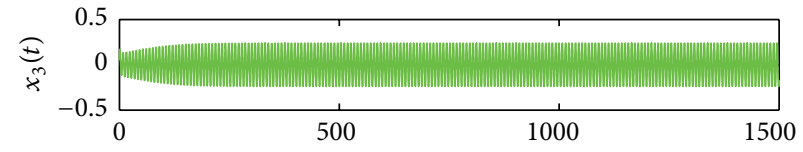

(c)

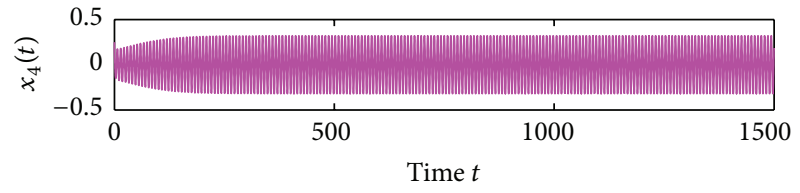

(d)

FIGURE 11: The trajectory of $x_{1}, x_{2}, x_{3}$, and $x_{4}$ when $\tau=0.85>$ $0.7915=\tau_{0}$.

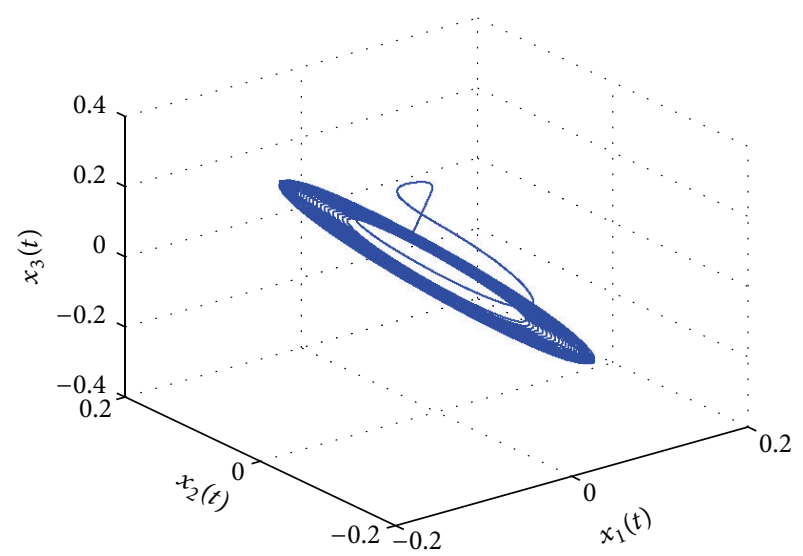

FIGURE 12: The phase plot of $x_{1}, x_{2}$, and $x_{3}$ when $\tau=0.85>0.7915=$ $\tau_{0}$.

unstable when $\tau_{2}>\tau_{2}^{*}$ and a Hopf bifurcation occurs, which can be illustrated by Figures 13, 14, 15, and 16.

\section{Conclusion}

In this paper, we have investigated a four-dimensional recurrent neural network with two discrete delays. Compared with the literature [11], we consider the neural network model which can reflect the really large neural networks more closely. By regarding the possible combinations of the two delays as the bifurcation parameter, sufficient conditions for the local stability of the zero equilibrium and the existence of Hopf bifurcation are obtained. If the conditions are satisfied, 


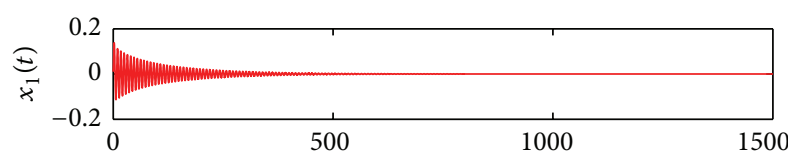

(a)

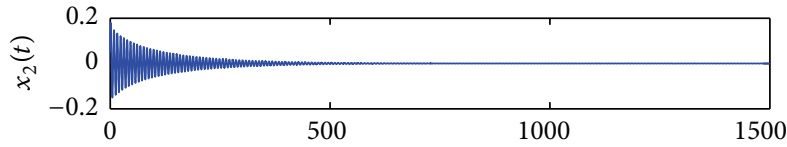

(b)

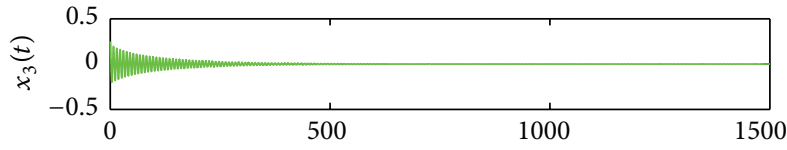

(c)

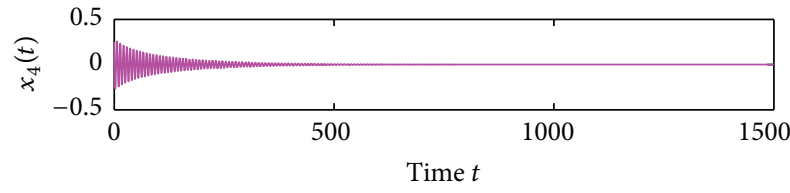

(d)

FIGURE 13: The trajectory of $x_{1}, x_{2}, x_{3}$, and $x_{4}$ when $\tau_{2}=1.65<$ $1.7488=\tau_{2}^{*}$ and $\tau_{1}^{*}=0.35$.

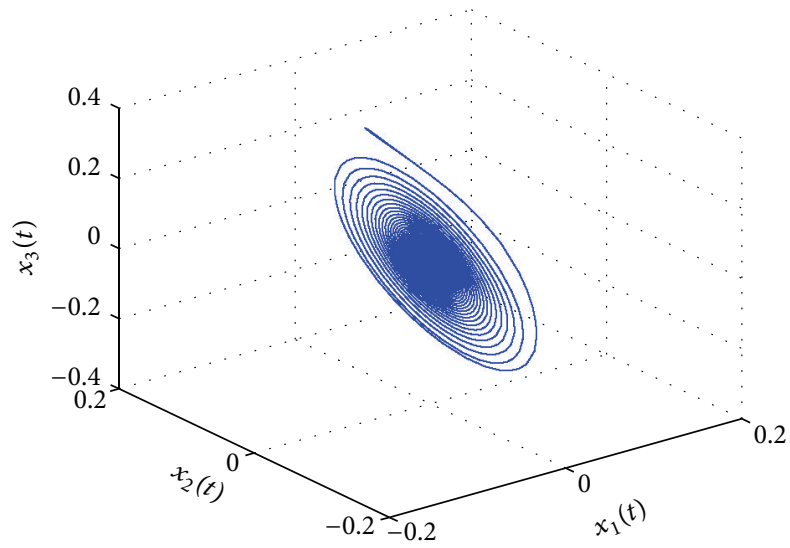

FIGURE 14: The phase plot of $x_{1}, x_{2}$, and $x_{3}$ when $\tau_{2}=1.65<$ $1.7488=\tau_{2}^{*}$ and $\tau_{1}^{*}=0.35$.

then there exists a critical value of the time delay below which the system is stable and above which the system is unstable. The results have shown that the two delays can play a complicated role on the model. And from the numerical simulations, we find that $\tau_{1}$ is marked in the model because the critical value of $\tau_{1}$ is much smaller than that of $\tau_{2}$ when we only consider them, respectively. Furthermore, the direction of the Hopf bifurcation and the stability of the bifurcating periodic solutions are discussed by the normal form theory and center manifold theory. Finally, some numerical simulations are also presented to support the theoretical analysis.

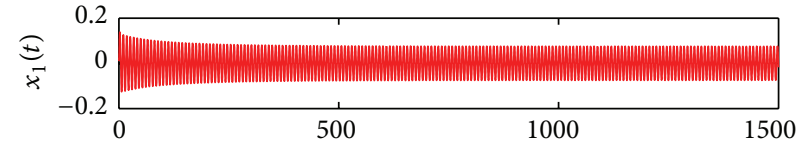

(a)

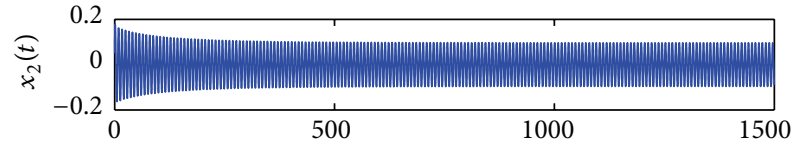

(b)

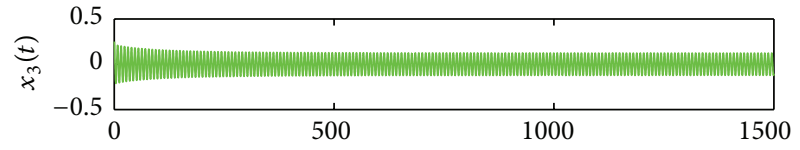

(c)

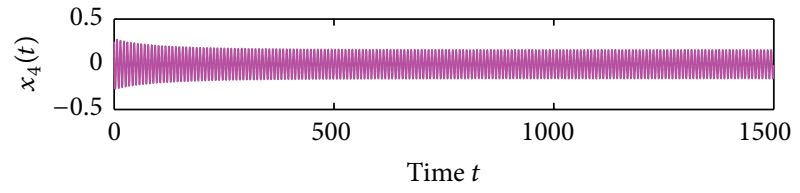

(d)

FIGURE 15: The trajectory of $x_{1}, x_{2}, x_{3}$, and $x_{4}$ when $\tau_{2}=1.85>$ $1.7488=\tau_{2}^{*}$ and $\tau_{1}^{*}=0.35$.

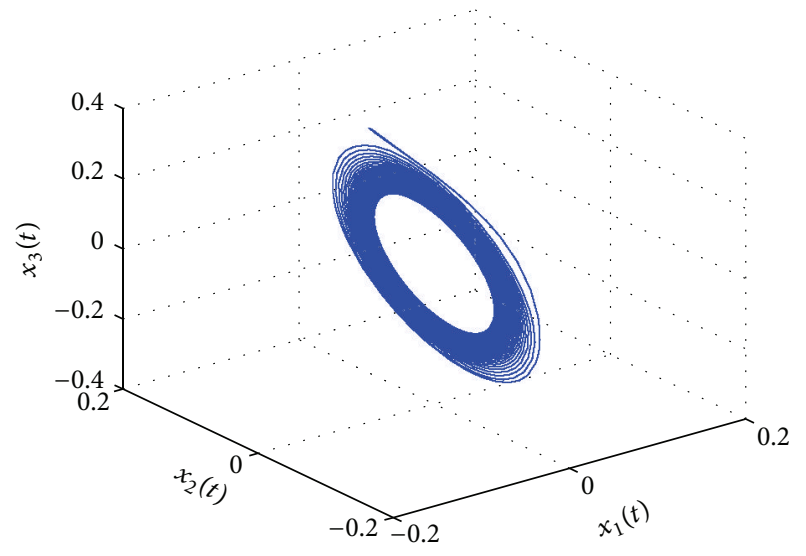

FIGURE 16: The phase plot of $x_{1}, x_{2}$, and $x_{3}$ when $\tau_{2}=1.85>$ $1.7488=\tau_{2}^{*}$ and $\tau_{1}^{*}=0.35$.

\section{Conflict of Interests}

The authors declare that there is no conflict of interests regarding the publication of this paper.

\section{Acknowledgments}

The authors are grateful to the two anonymous reviewers for their helpful comments and valuable suggestions on improving the paper. This work was supported by the National Natural Science Foundation of China (61273070), a project funded by the Priority Academic Program Development of Jiangsu Higher Education Institutions. 


\section{References}

[1] C. Yao, H. J. Chen, and J. P. Li, "Analysis of dynamic behaviors of improved pulse coupled neural network in image processing," Acta Automatica Sinica, vol. 34, no. 10, pp. 1291-1297, 2008 (Chinese).

[2] R. S. Toqeer and N. S. Bayindir, "Speed estimation of an induction motor using Elman neural network," Neurocomputing, vol. 55, no. 3-4, pp. 727-730, 2003.

[3] W.-M. Qi, Y.-C. Cheng, Q.-L. Ji, and W.-Y. Cai, "PID Elman neural network and its application to dynamical system identification," Control and Decision, vol. 20, no. 10, pp. 1197-1200, 2005 (Chinese).

[4] J. Yang, Y. Wen, and P. Li, "Feature extraction and identification of leak acoustic signal in water distribution pipelines using correlation analysis and approximate entropy," Chinese Journal of Scientific Instrument, vol. 30, no. 2, pp. 272-279, 2009 (Chinese).

[5] B. Luitel and G. K. Venayagamoorthy, "Quantum inspired PSO for the optimization of simultaneous recurrent neural networks as MIMO learning systems," Neural Networks, vol. 23, no. 5, pp. 583-586, 2010.

[6] L. Y. Wang, T. Y. Chai, and Z. Fang, "Neural network control and application of robotic manipulators including actuator dynamics," Acta Automatica Sinica, vol. 35, no. 5, pp. 622-626, 2009 (Chinese).

[7] Y. X. Wu and C. Wang, "Deterministic learning based adaptive network control of robot in task space," Acta Automatica Sinica, vol. 39, no. 1, pp. 1-10, 2013 (Chinese).

[8] J. Chen, Z. P. Li, and G. Z. Zhang, "Variable structure neural network adaptive robust control," Acta Automatica Sinica, vol. 36, no. 1, pp. 174-178, 2010 (Chinese).

[9] J. Wei and S. Ruan, "Stability and bifurcation in a neural network model with two delays," Physica D, vol. 130, no. 3-4, pp. 255-272, 1999.

[10] B. Wang and J. Jian, "Stability and Hopf bifurcation analysis on a four-neuron BAM neural network with distributed delays," Communications in Nonlinear Science and Numerical Simulation, vol. 15, no. 2, pp. 189-204, 2010.

[11] A. Hajihosseini, G. R. Rokni Lamooki, B. Beheshti, and F. Maleki, "The Hopf bifurcation analysis on a time-delayed recurrent neural network in the frequency domain," Neurocomputing, vol. 73, no. 4-6, pp. 991-1005, 2010.

[12] F. Maleki, B. Beheshti, A. Hajihosseini, and G. R. R. Lamooki, "The bogdanov-takens bifurcation analysis on a three dimensional recurrent neural network," Neurocomputing, vol. 73, no. 16-18, pp. 3066-3078, 2010.

[13] J. Wei and C. Zhang, "Bifurcation analysis of a class of neural networks with delays," Nonlinear Analysis, vol. 9, no. 5, pp. 2234-2252, 2008.

[14] C. Xu and X. He, "Stability and bifurcation analysis in a class of two-neuron networks with resonant bilinear terms," Abstract and Applied Analysis, vol. 2011, Article ID 697630, 21 pages, 2011.

[15] M. Xiao, W. X. Zheng, and J. D. Cao, "Bifurcation and control in a neural network with small and large delays," Neural Networks, vol. 44, pp. 132-142, 2013.

[16] C. Feng and R. Plamondon, "An oscillatory criterion for a time delayed neural ring network model," Neural Networks, vol. 2930, pp. 70-79, 2012.

[17] X. Yang, M. Yang, H. Liu, and X. Liao, "Bautin bifurcation in a class of two-neuron networks with resonant bilinear terms," Chaos, Solitons \& Fractals, vol. 38, no. 2, pp. 575-589, 2008.
[18] A. Ruiz, D. H. Owens, and S. Townley, "Existence, learning, and replication of periodic motions in recurrent neural networks," IEEE Transactions on Neural Networks, vol. 9, no. 4, pp. 651-661, 1998.

[19] S. Townley, A. Ilchmann, M. G. Weiss et al., "Existence and learning of oscillations in recurrent neural networks," IEEE Transactions on Neural Networks, vol. 11, no. 1, pp. 205-214, 2000.

[20] H. Hu and L. Huang, "Stability and Hopf bifurcation analysis on a ring of four neurons with delays," Applied Mathematics and Computation, vol. 213, no. 2, pp. 587-599, 2009.

[21] E. Javidmanesh, Z. Afsharnezhad, and S. Effati, "Existence and stability analysis of bifurcating periodic solutions in a delayed five-neuron BAM neural network model," Nonlinear Dynamics, vol. 72, no. 1-2, pp. 149-164, 2013.

[22] C. Xu, X. Tang, and M. Liao, "Stability and bifurcation analysis of a six-neuron BAM neural network model with discrete delays," Neurocomputing, vol. 74, no. 5, pp. 689-707, 2011.

[23] B. D. Hassard, N. D. Kazarinoff, and Y. H. Wan, Theory and Applications of Hopf Bifurcation, vol. 41, Cambridge University Press, Cambridge, UK, 1981.

[24] X.-Y. Meng, H.-F. Huo, and H. Xiang, "Hopf bifurcation in a three-species system with delays," Journal of Applied Mathematics and Computing, vol. 35, no. 1-2, pp. 635-661, 2011. 


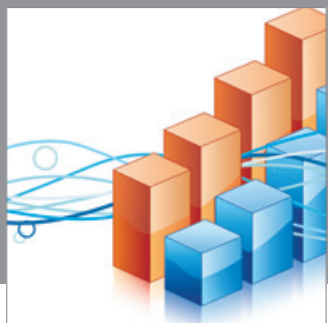

Advances in

Operations Research

mansans

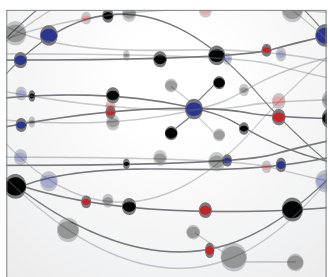

The Scientific World Journal
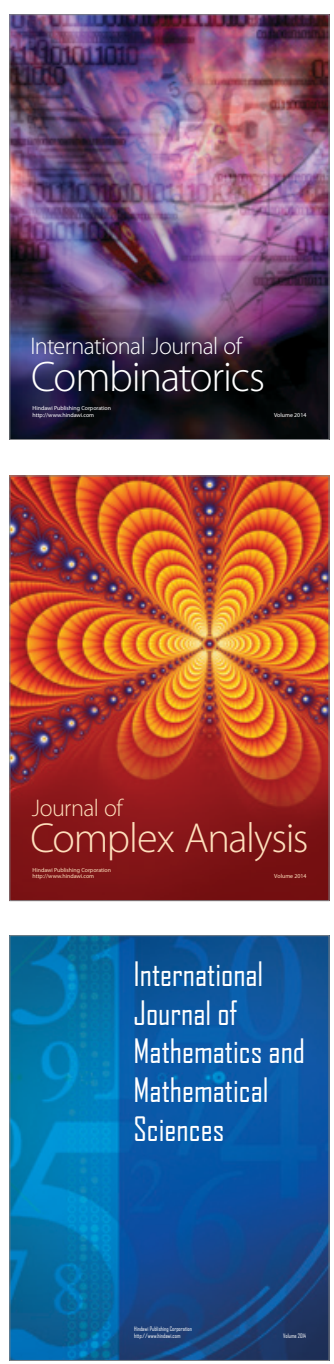
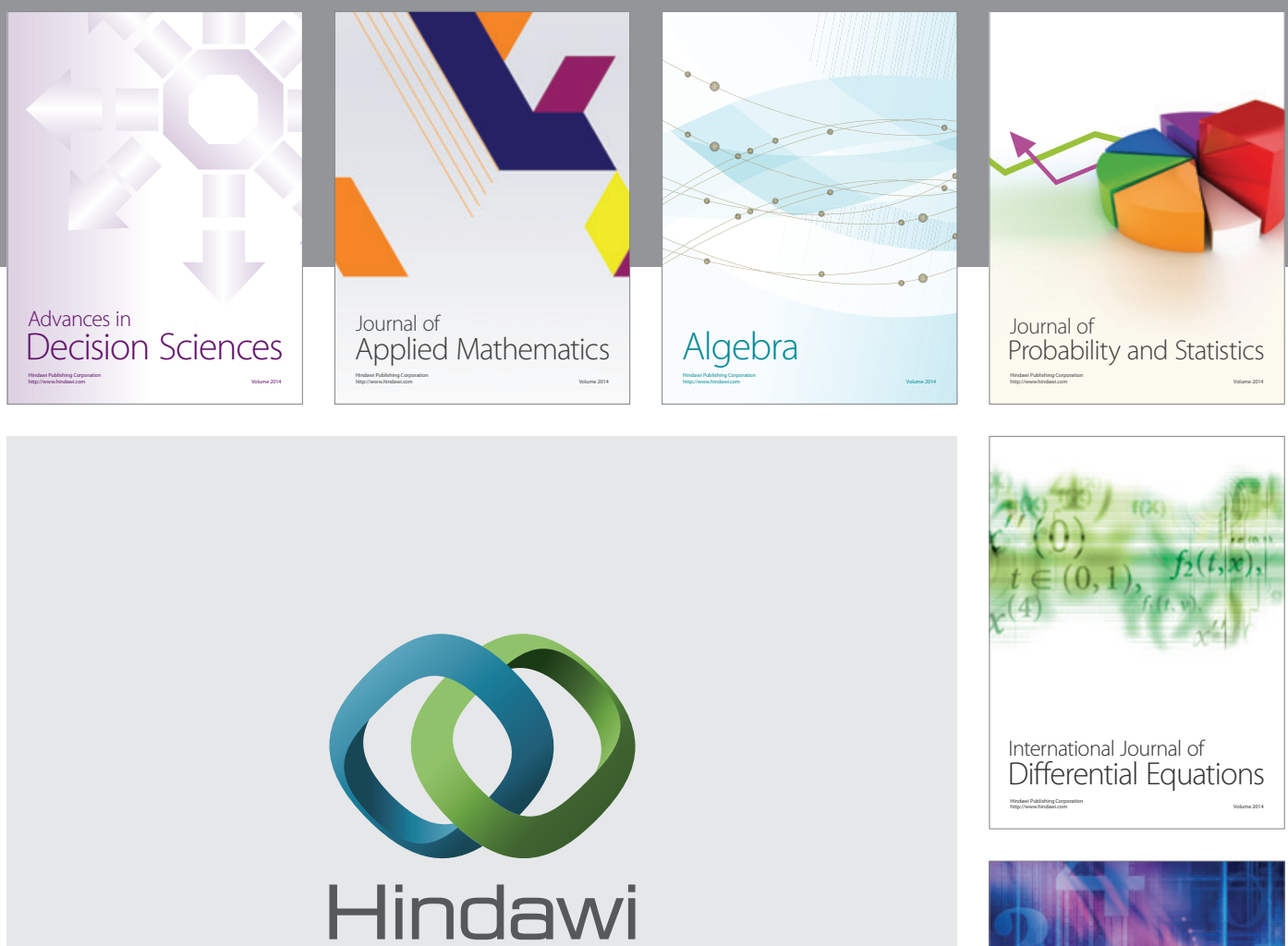

Submit your manuscripts at http://www.hindawi.com
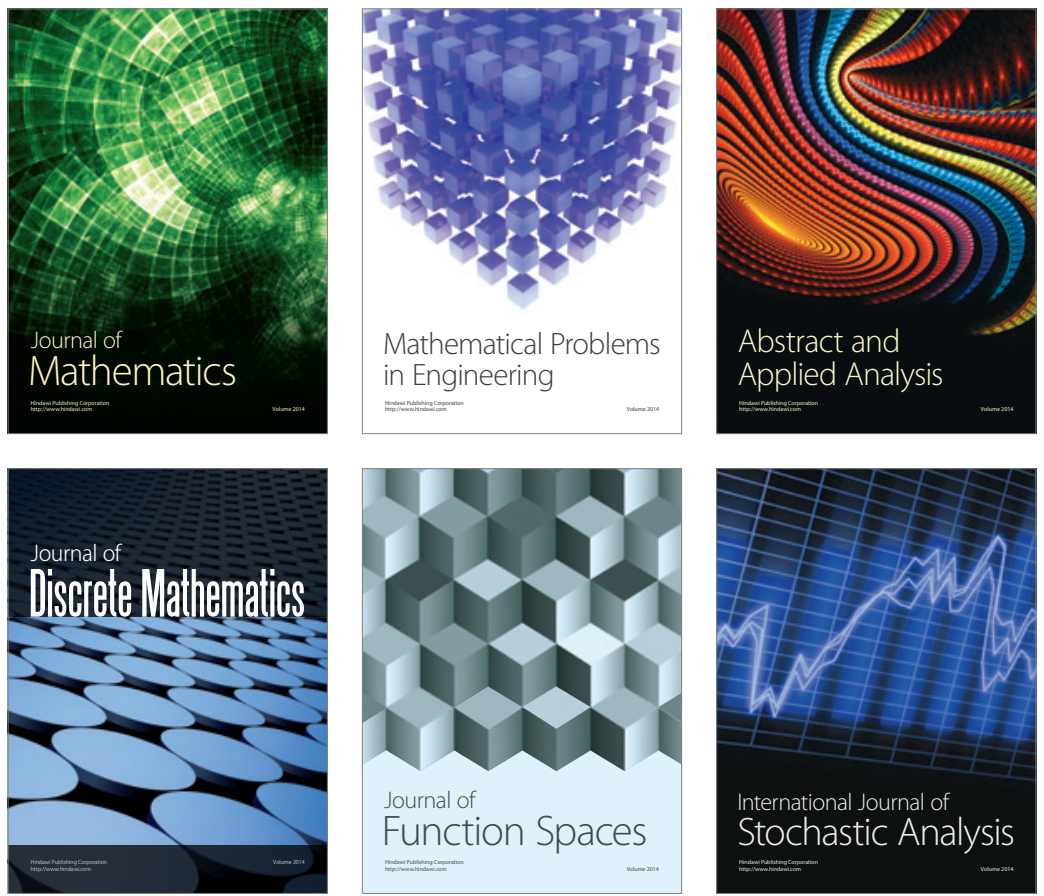

Journal of

Function Spaces

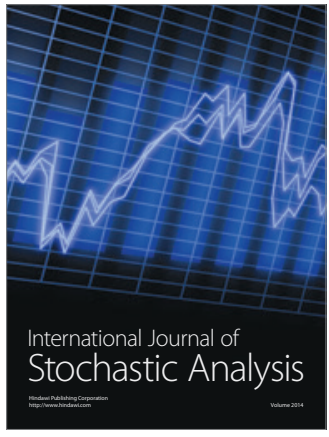

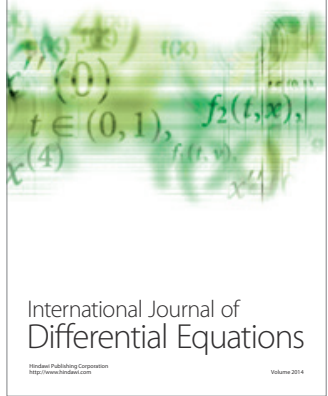
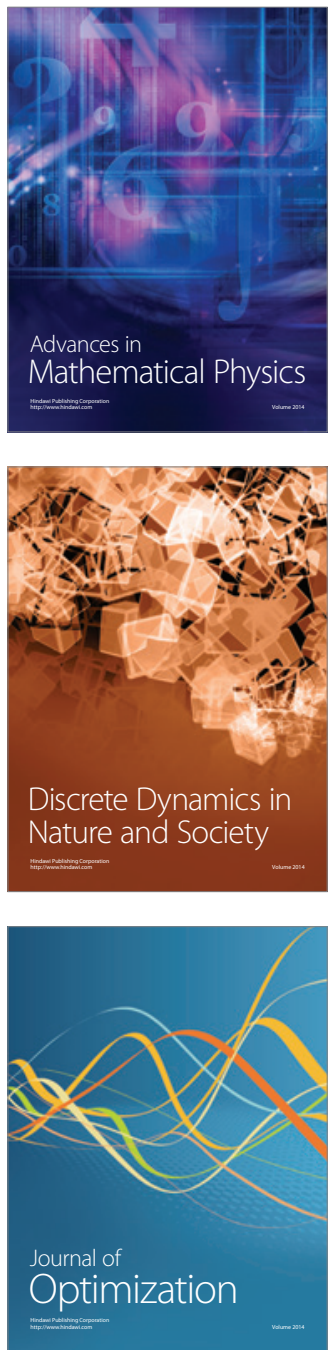